\title{
MedienPädagogik
}

Zeitschrift für Theorie und Praxis der Medienbildung

\section{Fallkonstellationen zum Einsatz digitaler Medien im Unterricht}

\section{Eine qualitative, lehrendenzentrierte Betrachtung}

\author{
Lukas Gerthofer und Jennifer Schneider
}

\section{Zusammenfassung}

Diesem Beitrag liegt der Gedanke zu Grunde, dass sich die Dominanz digitaler Medien im Alltag von Schülerinnen und Schülern nicht ausreichend in den Bildungseinrichtungen in Deutschland widerspiegelt. Dabei steht die Forschungsfrage im Mittelpunkt, welche Konstellationen von personenbezogenen Ursachen von Lehrpersonen zu (mangelndem) Einsatz digitaler Medien im Unterricht führen. Zur Beantwortung der zentralen Fragestellung wurde das auf den Bildungskontext adaptierte und bereits empirisch gut untersuchte UTAUT-Modell verwendet. Daraus ergeben sich im Wesentlichen sechs Einflussfaktoren: Der wahrgenommene Mehrwert für die Lehrpersonen, der vermutete Aufwand für die Integration der digitalen Medien in den Unterricht, die selbstbezogene Überzeugung die Medien auch im Unterricht (sicher) einsetzen zu können, die unterstützenden Bedingungen, die Einstellung der Lehrpersonen gegenüber Medien und der soziale Einfluss. Es wurden elf in die Untersuchung einfliessende Interviews mit Lehrpersonen an weiterführenden Schulen geführt und einer qualitativen Inhaltsanalyse unterzogen. Die Aussagen der Lehrpersonen wurden hinsichtlich der oben genannten Einflussfaktoren analysiert. Das daraus resultierende Muster der Einflussfaktoren auf die Mediennutzung wurde darauf aufbauend in einem fallstudienähnlichen Design beschrieben und veranschaulicht. Durch den qualitativen Ansatz ist es möglich, besser zu verstehen, in welchen Konstellationen Lehrpersonen Medien nutzen. 


\title{
Using digital media in the classroom. Case examples for predictor combinations. A qualitative and teacher centered examination
}

\begin{abstract}
This contribution is based on the idea that the dominance of digital media in pupils' everyday life is not sufficiently reflected in educational institutions in Germany. It is investigated which patterns of personal causes and factors of teachers lead to (neglecting) the use of digital media in class. To answer the central question an adapted form of the «Unified theory of acceptance and use of technologY» (UTAUT) is used as a framework. The UTAUT is widely used in educational contexts and empirically reviewed. Six influencing aspects are identified: The additional value for teachers when using digital media in class, the estimated effort for integrating digital media in lessons, the self-conviction and certitude of using media in class, the attitude of teachers concerning digital media and social influence. A qualitative content analysis of eleven interviews with teachers teaching at secondary schools was conducted. The insights gained from the interviews were traced back to the UTAUT model. The resulting pattern of personal factors influencing the use of media in class was described in a casestudy like setup and the usage of digital media in class illustrated. The qualitative design allows a better understanding in which constellations teachers use media in class.
\end{abstract}

\section{Die Mediatisierung der Schule - warum Medien Einzug ins Klassenzimmer finden sollen}

Unser gesellschaftliches Miteinander ist durch eine zentrale Rolle digitaler Medien geprägt (Kommer und Biermann 2012, 82), was sich nicht zuletzt durch die Bezeichnung der Medien- und Informationsgesellschaft manifestiert, in der wir leben (Hoffmann und Mikos 2007, 7). Unter dem Begriff der digitalen Medien finden sich sämtliche Techniken, Geräte und Systeme wieder, welche den Informations- und Kommunikationstechnologien (IKT) zuzuordnen sind (Tappe 2019, 1002). Im (ausserschulischen) Alltag der Schülerinnen und Schüler sind die IKT nicht mehr wegzudenken, was sich 
unter anderem am Faktum zeigt, dass bei nahezu allen Jugendlichen in Deutschland das Smartphone zum täglichen Begleiter gehört (Feierabend, Plankenhorn, und Rathgeb 2017, 9 ff.). Dem gegenüber stehen die Ergebnisse der aktuellen «International Computer and Information Literacy Study» (ICILS) aus 2018, welche einen internationalen Vergleich der Kompetenzen von Lernenden in der achten Klasse darstellt, wonach lediglich ein Viertel der befragten Schülerinnen und Schüler hierzulande mindestens einmal in der Woche digitale Medien in der Schule zu schulbezogenen Zwecken einsetzen (Eickelmann et al. 2019, 19). Auch im Vergleich zu den anderen Teilnehmerländern werden in Deutschland digitale Medien im Unterricht unterdurchschnittlich häufig genutzt. Neben den fehlenden technischen Rahmenbedingungen (z. B. W-LAN-Zugang) gehören unter anderem auch personenbezogene Faktoren der Lehrpersonen zu den Gründen des weniger häufigen Medieneinsatzes (Eickelmann et al. 2019, 14). Nur etwa ein Drittel der befragten Lehrenden sehen im digitalen Medium das Potential zur Verbesserung schulischer Leistungen (Eickelmann et al. 2019, 18). Zusammenfassend konstatiert Tappe (2009, 1005), gebe es eine Diskrepanz zwischen den Alltagserfahrungen der Lernenden und der schulischen Lernwirklichkeit.

Neben der fehlenden Wahrnehmung der Sinnhaftigkeit des Medieneinsatzes werden in der Literatur weitere lehrendenbezogene Faktoren genannt, die den Medieneinsatz hemmen. Hierzu zählen beispielsweise der Habitus der Lehrenden, der sich in Vorbehalten gegenüber dem Einsatz digitaler Medien manifestiert (Schiefner-Rohs, Gomez Tutor, und Menzer 2015, 31) oder die Wahrnehmung des mit dem Einsatz zunehmenden zeitlichen Mehraufwands (Lermen 2008, 248). Als ein Teilergebnis einer $\mathrm{Me}$ tastudie mit über 125 Studien weltweit fasst Waffner (2020, 71 ff.) die Bedingungen für den Einsatz der Medien zusammen und bestätigt, dass neben der Grundvoraussetzung des Vorhandenseins der technischen Infrastruktur auch die organisatorischen Gegebenheiten relevant sind. Waffner $(2020,76)$ resümiert, dass die Nutzung der Medien in hohem Masse von der einzelnen Lehrkraft abhänge und aus diesem Grund 
«[...] ist es von besonderem Interesse, die bildungswissenschaftliche Forschung zu Einstellungen, der pädagogischen Haltung des Lehrpersonals, vorhandenen und noch notwendigen Kompetenzen sowie die Frage des Kompetenzerwerbs in Fort- und Weiterbildungen zu ermitteln.»

Es bleibt trotz der zahlreichen Studien zum Thema Mediennutzung im Unterricht zu konstatieren, dass die Gründe für eine geringe Nutzung in Deutschland noch nicht abschliessend beurteilt werden können und Ursachen für oder gegen Mediennutzung nicht durchgehend in ihrer Gesamtwirkung gewürdigt werden.

Als Beitrag zur Schliessung der Lücke wird als Ziel der vorliegenden Untersuchung die Ergründung des Medieneinsatzes durch Lehrende im Sekundarbereich ${ }^{1}$ an deutschen Schulen in Bezug auf lehrendenbezogene Aspekte ausgegeben. Konkret wird folgender Frage nachgegangen:

Welche Konstellationen von personenbezogenen Ursachen führen zu (mangelndem) Einsatz digitaler Medien im Unterricht?

Als Rahmen und zum Zwecke der Systematisierung der zahlreichen Faktoren, welche auf die Mediennutzung wirken, wird das Modell der «Unified theory of acceptance and use of technology» (UTAUT) (Venkatesh, Thong, und $\mathrm{Xu}$ 2012, 178) als Teil der Technologieakzeptanzforschung verwendet. UTAUT stellt im Wesentlichen die Kausalzusammenhänge (Nistor, Wagner, und Heymann 2012, 350) jener personenbezogenen Einflussfaktoren dar, welche der Absicht und der tatsächlichen Nutzung neuer Technologien vorangehen. Dieses Modell kann, wie bereits in einigen Studien geschehen, auf den Bildungskontext übertragen werden und stellt Prädiktoren für die tatsächliche Nutzung von Medien im Unterricht dar. Das Modell ist empirisch bereits gut überprüft (Tappe 2019, 1008) und gilt als robust im Sinne der medienpädagogischen Forschung (Nistor, Wagner, und Heymann 2012, 353).

Im weiteren Verlauf werden der Forschungsstand zum UTAUT-Modell und die von der Mediennutzung unabhängigen Faktoren dargestellt um darauf aufbauend das Forschungsdesign des Beitrags darzustellen. Der methodische Kern ist ein fallstudienartiges Design durch elf qualitative

1 Die sekundare Stufe umfasst alle Schularten ab der 5. Klasse. 
Interviews mit Lehrpersonen der Sekundarstufe. Die Ergebnisse der Interviews dienen der Veranschaulichung der unabhängigen Faktoren und der Erhebung von Fallkonstellationen, die zur Nutzung oder Meidung digitaler Medien im Unterricht führen. Abschliessend erfolgt eine Diskussion der Ergebnisse.

\section{Forschungsstand zu den beeinflussenden Faktoren auf den Einsatz von Medien im Unterricht}

\subsection{Unified Theory of Acceptance and Use of TechnologY (UTAUT)}

Technologieakzeptanz ist trotz der Nachteile solcher Modelle (Nistor, Wagner, und Heymann 2012, 351 f.) ein gängiger Rahmen, der sowohl im deutschsprachigen als auch im internationalen Raum als Erklärungsansatz für die Nutzung von Medien im Unterricht genutzt wird. Über die Jahre hinweg sind einige Technology Acceptance Models (TAM) entstanden, welche Prädiktoren der jeweiligen Einstellungs- und Nutzungsakzeptanz von Technologien beschreiben (Bach 2016, 114). 2003 haben Venkatesh, Morris, Davis und Davis $(2003,425)$ Modelle mehrere Forscher aus dem TAM Kontext durch einen umfassenden Literatur-Review zusammengeführt und dabei das UTAUT entwickelt. Dieses Modell beschreibt mehrere Faktoren, welche Einfluss auf die Nutzungsabsicht einer Person zum Einsatz einer Technologie haben, die wiederum der tatsächlichen Nutzung vorangeht (Venkatesh, Thong, und Xu 2012, 178).

Wird das UTAUT zur Untersuchung des Medieneinsatzes herangezogen, bedarf es einer entsprechenden Abänderung im Hinblick auf den Kontext der Lehrenden. Hierbei wird die Annahme getroffen, dass sich dieses Modell als Grundlage zur Untersuchung der Akzeptanz Lehrpersonen gegenüber dem Einsatz digitaler Medien im Unterrichtsgeschehen hinreichend eignet. Dies begründet sich insbesondere in der bereits erfolgten Adaption der TAM-Modelle auf die Bildungstechnologien (für Deutschland: Tappe 2019, Bach 2016, Prasse 2012, Nistor, Wagner und Heymann 2012). Das UTAUT-Modell wurde bereits in mehreren Beiträgen als theoretisches 
Rahmenmodell für die Erforschung der Technologieakzeptanz im Bildungsbereich berücksichtigt (beispielsweise Perienen 2020, Ameri et al. 2019, Garone et al. 2019, Sumak und Sorgo 2016, ein Literatur-Review zur Anwendung von UTAUT ist in Williams, Rana und Dwivedi 2015 für Beiträge bis $2011 \mathrm{zu}$ finden; Eder 2015, Lin, Lu, und Shang-Chia 2013, Nistor, Wagner, und Heymann 2012, Birch und Irvine 2009).

Das UTAUT-Modell ist einfach aufgebaut und schliesst von Einflussfaktoren, die wiederum in Kategorien eingeteilt werden (die abhängigen Variablen), auf die Nutzungsabsicht der Technologie und daraus wiederum auf die Nutzung der Technologie (die unabhängige Variable). Der Beitrag von Tappe (2019) stellt ein auf den Bildungsbereich angepasstes Modell dar, das auf bereits auf den Bildungsbereich angepasste UTAUT-Modelle basiert. Tappe erweitert das Modell um Variablen, die gemäss dem aktuellen Forschungsstand bei Lehrpersonen berücksichtigt werden sollten und im ursprünglichen Modell von Venkatesh (2013) verworfen wurden. Die ergänzten Variablen sind laut Tappe $(2019,1014)$ für Lehrpersonen relevant, da sie affektive Bereiche berücksichtigen, die wiederum für Lehrende wichtig sind.

Die Kategorien, die direkt auf die Nutzungsabsicht oder die tatsächliche Nutzung von Medien wirken, sind der erwartete Mehrwert, der geschätzte Aufwand, der soziale Einfluss und die unterstützenden Bedingungen (s. Abbildung 1). Bezogen auf den Kontext der Medienintegration in den Unterricht im Sinne dieses Beitrags können diese wie folgt eingegrenzt werden (Venkatesh, Morris, Davis, und Davis 2003, 446 ff., Tappe 2019, 1007 f., Übertragung auf den Medienkontext durch den Autor und die Autorin des vorliegenden Beitrags):

- Erwarteter Mehrwert: Wird die Medienintegration im Unterricht didaktisch oder arbeitsorganisatorisch als vorteilhaft betrachtet?

- Geschätzter Aufwand: Wie hoch wird der Aufwand zum Erwerb einer Medienkompetenz und zur Nutzung der Medien im Unterricht eingeschätzt?

- Sozialer Einfluss: Gibt es externe, d.h. ausserhalb der Schule liegende, Einflüsse, die auf die Nutzung von Medien im Unterricht Einfluss haben könnten, wie beispielsweise Meinungen von Eltern? 
- Unterstützende Bedingungen: Werden durch die Lehrkraft Unterstützung oder Vorschriften zur Medienintegration wahrgenommen, beispielsweise durch die Schulleitung oder das Kollegium?

Die Moderatorenvariablen, also solche, die wiederum auf die oben vorgestellten direkt beeinflussenden Variablen einwirken, sind im angepassten Modell laut Tappe (2019, 1014 f.), die folgenden:

- Selbstbezogene Überzeugung/Kenntnisse und Sicherheit: Wie schätzt die Lehrperson die eigenen Kenntnisse und Sicherheit in Bezug auf eine Mediennutzung im Unterricht ein?

- Einstellung zur Technologienutzung/Medialer Habitus²: Wie ist die Grundeinstellung der Lehrperson gegenüber der Verwendung von $\mathrm{Me}$ dien (sowohl privat als auch bezogen auf die Nutzung im Unterricht)?

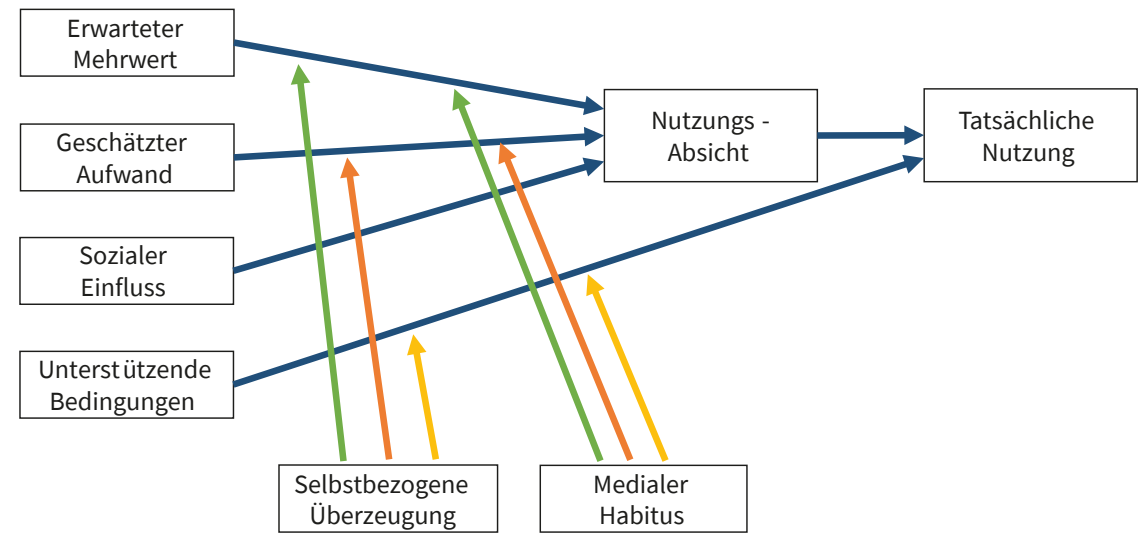

Abb. 1.: Modell der Akzeptanz zum Einsatz digitaler Medien in Anlehnung an Tappe (2019, 1007).

2 Tappe (2019) unterscheidet zwischen der positiven Grundeinstellung zur Mediennutzung im privaten und im unterrichtlichen Bereich auf der einen Seite und einer negativen Affektivität der Mediennutzung im Unterricht auf der anderen Seite. Da diese Aspekte zwei Seiten derselben Medaille sind, werden die Faktoren im vorliegenden Beitrag nicht getrennt voneinander betrachtet. 


\subsection{Erwarteter Mehrwert}

Der erwartete Mehrwert dient dem Modell nach zur Feststellung, inwieweit die Nutzung digitaler Medien zu einer didaktischen und arbeitsorganisatorischen Leistungssteigerung führt (Bach 2016, 117).

Mehrere Studien in jüngerer Vergangenheit zeigen, dass deutsche Lehrpersonen grosse Vorbehalte gegenüber digitalen Medien haben (SchiefnerRohs, Gomez Tutor, und Menzer 2015, 19). Dabei kommt häufig die (vermeintliche) These der geringen Zweck- und Sinnhaftigkeit zum Tragen, welche Lehrende vom Einsatz digitaler Medien im Unterricht abhalten könnten (Breiter, Stolpmann, und Welling 2010, 7). Zur Sinnhaftigkeit stellt sich dabei die Frage nach dem Mehrwert der digitalen Medien gegenüber herkömmlichen Medien, sprich an welcher Stelle der Einsatz digitaler $\mathrm{Me}$ dien pädagogische und didaktische Potentiale bietet (Lermen 2008, 244). Auffällig ist auch die verhaltene Einschätzung der Lehrpersonen, welche der ICILS 2018 nach in nur 34,7 \% der Fälle angaben, digitale Medien hätten Potentiale zur Verbesserung schulischer Leistungen (Eickelmann et al. 2019, 18). In einem Beitrag von Kommer wird als allgemeines Ergebnis angeführt, dass den digitalen Medien implizit ein gewisser Bildungswert abgesprochen wird (Kommer 2016, 60).

\subsection{Geschätzter (zusätzlicher) Aufwand}

Beim geschätzten Aufwand wird die Einschätzung betrachtet, inwiefern die Nutzung digitaler Medien im Unterricht und der Erwerb von Medienkompetenzen als einfach empfunden werden (Bach 2016, 117).

In diesem Kontext ist insbesondere der wahrgenommene Aufwand zur Erlernung von Kompetenzen im Umgang mit digitalen Medien sowie deren Einsatz im Unterricht gemeint. Beispielsweise lässt sich dazu der zeitliche Mehraufwand nennen, welchen Lehrende bei der Aneignung ihrer Medienkompetenzen auf sich zukommen sehen (Lermen 2008, 248). Gysbers fasst als Fazit seiner Studie unter anderem zusammen, dass Lehrpersonen die Medienbildung als zusätzliche Belastung empfinden, welche über das normale Arbeitspensum hinaus zusätzlich erbracht werden müsste (Gysbers 2008, 198). 


\subsection{Sozialer Einfluss}

Der Gedanke hinter dem sozialen Einfluss im UTAUT-Modell stellt die Wahrnehmung einer Person dar, in welchem Masse ihr persönliches Umfeld eine Mediennutzung erwartet (Nistor, Wagner, und Heymann 2012, 349). Das bedeutet auf den Bildungskontext übertragen, dass sich die Erwartungen der Eltern, des Lehrerkollegiums und auch der Lernenden in Bezug auf die Medienintegration durch den Lehrenden auswirken können.

\subsection{Unterstützende Bedingungen}

Die unterstützenden Bedingungen wirken sich dem Modell nach nicht auf die Nutzungsabsicht, sondern vielmehr auf die tatsächliche Nutzung aus, wenn eine Absicht bereits besteht. Dies begründet Venkatesh (2003, 465 f.) damit, dass sich die Effekte der unterstützenden Bedingungen mit dem Faktor des erwarteten Mehrwertes überlagern und damit bereits indirekt auf die Nutzungsabsicht Einfluss nehmen. Inwieweit eine Person die existierenden organisatorischen und technischen Ressourcen wahrnimmt, wird anhand dieses Faktors gemessen (Venkatesh 2003, 460). Zu den technischen Rahmenbedingungen zählen alle Ressourcen einer schulischen Medienausstattung, welche den Einsatz digitaler Medien im Unterricht ermöglichen. Darunter fallen auch alle Aspekte des Umfeldes eines Lehrenden in der Schule, beispielsweise der Austausch im Kollegium oder die Gestaltungsfreiheit im Hinblick auf den Einsatz digitaler Medien. Untersuchungen des Instituts für Informationsmanagement Bremen zeigen, dass die Schulleitung eine zentrale Rolle bei der Medienintegration darstellt (Breiter, Stolpmann, und Welling 2010, 11). Sie fördert beispielsweise die Strukturen für die Kommunikation und den Austausch im Kollegium, was zu einem positiven Effekt der Medienintegration führen kann (Breiter, Stolpmann, und Welling 2010, 11; Brandhofer 2015, 152).

\subsection{Selbstbezogene Überzeugung/Kenntnisse und Sicherheit} Im auf den Bildungsbereich angepassten UTAUT-Modell finden sich die Aspekte der Kenntnisse und Sicherheit wieder, welche sich auf den Mehrwert, den Aufwand und die unterstützenden Bedingungen auswirken können. 
Dies liegt darin begründet, dass Lehrende den Einsatz digitaler Medien aufgrund der vorherrschenden Unsicherheit oder Unkenntnis mit einem geringen Mehrwert oder hohen Aufwand assoziieren könnten. Zudem ist es möglich, dass Lehrpersonen durchaus die Absicht haben, digitale $\mathrm{Me}$ dien in ihren Unterricht einzusetzen, dies aber letztlich aufgrund mangelnder Kenntnisse oder Sicherheiten nicht umsetzen. Demnach wird die Wahrnehmung auf die unterstützenden Bedingungen durch die Variable der Kenntnisse und Sicherheit beeinflusst, indem die vorhandenen Möglichkeiten durch das Wissen über digitale Medien unterschiedlich wahrgenommen werden.

Als zentrale Barriere der Mediennutzung erkennen Schulz-Zander und Eickelmann $(2008,6)$ aus verschiedenen Untersuchungen die mangelnden Kenntnisse und Fertigkeiten der Lehrpersonen im Umgang mit digitalen Medien. Auch weitere Autoren kommen zu dem Schluss, dass die Verwendung digitaler Medien im Fachunterricht von der Vertrautheit der Lehrenden mit Computern und dem Internet in deren Alltag zusammenhängt (Grasmück, Büttner, und Vollmeyer 2010, 274).

Zudem wird der Einsatz digitaler Medien im Unterricht durch Lehrende des Öfteren mit dem Informatikunterricht verwechselt (Brandhofer 2015, 49). Möglicherweise wird durch die Einstellung der Lehrpersonen, keinen Informatikunterricht halten zu können, die Unsicherheit im Umgang mit diesen Medien weiter verstärkt. Ein Artikel von Knaus und Valentin zeigt, dass diese Reserviertheit unter anderem auf die Unkenntnis des sinnvollen Einsatzes von Medien im Unterricht zurückzuführen ist (Knaus und Valentin 2016, 160). Die Befürchtung der Lehrenden, mit den technischen Entwicklungen nicht Schritt halten zu können, stellt ein weiteres mögliches Hindernis zum Einsatz digitaler Medien im Unterricht dar (Gysbers 2008, 191). Kommer und Biermann kommen in ihren qualitativen Untersuchungen zum Ergebnis, dass Studierende im Lehramt ihre Medienkompetenzen gegenüber dem Computer als gering einschätzen, was die Angst vor dem Scheitern in Unterrichtssituationen und der damit verbundenen Blamage gegenüber den Lernenden schürt (Kommer und Biermann 2012, 101). 


\subsection{Grundeinstellung/Habitus der Lehrenden gegenüber digitalen Medien}

Das UTAUT-Modell wird weiter durch den Einbezug des medialen Habitus ergänzt. Dieser kann nicht als Ersatz zum sozialen Einfluss angesehen werden, da mit dem sozialen Habitus die Grundhaltung der Lehrenden und damit die inneren Motive selbst gemeint sind und nicht die externen Einflüsse. Den medienbezogenen Habitus erwirbt jeder Mensch durch seine Entwicklung im sozialen Raum und seiner medialen Umwelt (Kommer und Biermann 2012, 90), sprich seinem Elternhaus, seinen Erfahrungen im Umgang mit Medien sowie den Meinungen in seinem Umfeld. Damit könnte die grundlegende Haltung der Lehrperson gegenüber digitalen Medien eine beeinflussende Variable für den erwarteten Mehrwert und Aufwand sowie die Wahrnehmung der unterstützenden Bedingungen sein. Zur Ergründung möglicher Ursachen zeigt Kommer $(2016,60)$, dass dieses Phänomen besonders in Deutschland zum Tragen kommt. So ist der mediale Habitus angehender Lehrpersonen durch Reste bürgerlichen Ressentiments gegenüber digitalen Medien geprägt (Schiefner-Rohs, Gomez Tutor, und Menzer 2015, 19), welche sich beispielsweise in der differierenden Wertigkeit von Medien zeigt (lieber das Buch als digitale Medien).

Unter Berücksichtigung der in Kapitel 2 dargelegten Einflussfaktoren auf die Mediennutzung soll im weiteren Verlauf in einem fallstudienartigen Design über qualitative Interviews veranschaulicht werden, wie die Einflussfaktoren zusammenwirken und schlussendlich zur Medien(-nicht) nutzung führen.

\section{Das Forschungsdesign}

Es gilt zu veranschaulichen, warum Lehrpersonen Medien im Unterricht (nicht) einsetzen und welche Muster dabei zu identifizieren sind. Eine Veranschaulichung und erklärende Aspekte lassen sich durch quantitative Designs nur bedingt herstellen, weshalb auf qualitative Interviews mit Lehrpersonen der Sekundarstufe zurückgegriffen wird. 
Die Interviews haben einen fallstudienähnlichen und explanativen Charakter im Sinne von Yin (2003, 13), da Gegebenheiten der Mediennutzung im realen Kontext untersucht werden und das Gesamtbild des Beitrags einzelner Faktoren zur Mediennutzung noch nicht vollständig durch die Forschung geklärt ist. Das gegebene Ziel des Beitrags, die Veranschaulichung der Situation im realen Kontext und dem Aufzeigen von Kausalzusammenhängen in Bezug auf die Mediennutzung spricht für die Nutzung als Fallstudie (Yin 2003, 15).

Die Formen der empirischen Sozialforschung, welche mehrheitlich die Befragung als Datenerhebungsmethode verwenden, haben allesamt zum Ziel, Antworten auf die zugrundeliegenden Hypothesen zu finden (Atteslander und Cromm 2010, 123); ebenso setzt die Fallstudie laut Yin $(2003,22)$ eine Hypothesenbildung voraus.

\subsection{Hypothesen}

Die aufzustellenden Hypothesen ergeben sich konsequenterweise aus den im Forschungsüberblick dargestellten Erkenntnissen und dem durch empirische Studien bestätigten UTAUT-Modell. Zusammengefasst ergeben sich folgende Thesen:

- Lehrpersonen, die Medien häufig einsetzen, erwarten einen Mehrwert aus der Nutzung im Unterricht, schätzen einen geringeren Aufwand, berücksichtigen einen externen Einfluss im Sinne einer Erwartungshaltung, nehmen unterstützende Bedingen wahr, schätzen ihre Kenntnisse und Fertigkeiten in Bezug auf die Mediennutzung im Unterricht gut ein und haben eine positive Grundeinstellung zu Medien.

- Entsprechend schätzen Lehrpersonen, die Medien nur selten nutzen, die genannten Aspekte umgekehrt ein.

\subsection{Datenerhebung und -Auswertung}

Zur empirischen Datenerhebung wurde das Experteninterview gewählt. Das Gespräch wird als leitfadengestütztes Interview geführt, wobei dieser zur unbürokratischen und lockeren Führung des Interviews beiträgt (Meuser und Nagel 1991, 449). An dieser Stelle sei erwähnt, dass auch 
vereinzelt Interviews mit zwei sich bekannten Lehrpersonen gleichzeitig geführt werden. Dies soll insbesondere der Reichhaltigkeit an Antworten und Aussagen zugutekommen, da sich gelegentlich Meinungen erst bilden, wenn diese in der Diskussion angesprochen werden und die vertrauten Teilnehmer aufgrund der Atmosphäre eher «aus dem Nähkästchen» plaudern und damit Einstellungen transparenter werden (Vogl 2014, 581 f.). Alle Gespräche werden persönlich vor Ort geführt und aufgezeichnet, um im Nachgang die Gespräche transkribieren und auswerten zu können.

Der Leitfaden für die Interviews ist im Anhang des Beitrags dargestellt. Die Informationsbedarfe in Bezug auf die Interviews bestehen zum einen darin, die personenbezogenen Einflussfaktoren gem. UTAUT-Modell zu kategorisieren, um daraus wiederum Fallkonstellationen ermitteln zu können. Zum anderen sollen aus den Interviews Veranschaulichungen und Erklärungen aus Selbsteinschätzungen der Lehrpersonen gewonnen werden. Mit den Veranschaulichungen soll ein tieferes Verständnis für die Gründe des Handelns der Lehrpersonen gewonnen werden, die über die Korrelationen in quantitativen Studien hinausgehen. Weiterhin können ggf. über das Modell hinausgehende Faktoren, die in den Interviews genannt werden, identifiziert werden.

Bei der Auswahl der Stichprobe wurde darauf geachtet, dass unterschiedliche Rollen in der schulischen Lehre repräsentiert sind. Die Auswahl der Akteure ist aus praktischen Gründen auf Schulen in BadenWürttemberg begrenzt, wobei hier einige Schulleitungen um Mithilfe und Nennung von Lehrpersonen zur Durchführung der Untersuchung gebeten wurden. Bei der letztlichen Festlegung der Interviewpartner ist auf eine hinreichende Medienausstattung in den dortigen Klassenräumen geachtet worden, um äussere Einflüsse wie eine fehlende Medienausstattung als Grund für die Nichtnutzung von Medien auszuschliessen. Weiterhin wurde zu einem gewissen Grad auf eine hinreichende Diversifikation bezogen auf Geschlecht und Alter geachtet. Die Interviews wurden im März und April 2018 geführt.

Nachfolgende Tabelle stellt die Übersicht der interviewten Lehrpersonen dar. 


\begin{tabular}{|c|c|c|c|c|c|}
\hline Interviewte & $\begin{array}{l}\text { ID- } \\
\text { IP }\end{array}$ & Alter & Schulart & $\begin{array}{l}\text { Position und Unterrichts- } \\
\text { fächer }\end{array}$ & $\begin{array}{l}\text { Länge } \\
\text { (min.) }\end{array}$ \\
\hline Partnerin $\mathrm{A}^{3}$ & IP-A & $45-55$ & $\begin{array}{l}\text { Gemeinschafts- } \\
\text { schule }\end{array}$ & Schulleiterin; Deutsch & \multirow[t]{2}{*}{50} \\
\hline Partner B & IP-B & $25-35$ & $\begin{array}{l}\text { Gemeinschafts- } \\
\text { schule }\end{array}$ & $\begin{array}{l}\text { Klassenlehrer; Informatik, } \\
\text { Chemie }\end{array}$ & \\
\hline Partnerin C & IP-C & $25-35$ & $\begin{array}{l}\text { Gemeinschafts- } \\
\text { schule }\end{array}$ & $\begin{array}{l}\text { Klassenlehrerin; Deutsch, } \\
\text { Englisch }\end{array}$ & 20 \\
\hline Partnerin D & IP-D & $45-55$ & Realschule & $\begin{array}{l}\text { Klassenlehrerin, Englisch, } \\
\text { Kunst }\end{array}$ & 55 \\
\hline Partner E & IP-E & $35-45$ & Realschule & $\begin{array}{l}\text { Klassenlehrer; Mathe, } \\
\text { Gemeinschaftskunde }\end{array}$ & \multirow[t]{2}{*}{45} \\
\hline Partner F & IP-F & $45-55$ & Realschule & Fachlehrer; Deutsch & \\
\hline Partner G & IP-G & $25-35$ & Realschule & $\begin{array}{l}\text { Fachlehrer; Sport, Tech- } \\
\text { nik }\end{array}$ & 50 \\
\hline Partnerin $\mathrm{H}$ & IP-H & $55-65$ & Gymnasium & $\begin{array}{l}\text { Fachlehrerin; Geografie, } \\
\text { Geschichte, Politik }\end{array}$ & 25 \\
\hline Partner I & IP-I & $45-55$ & Gymnasium & $\begin{array}{l}\text { Fachlehrer; Deutsch, Ge- } \\
\text { schichte, Gemeinschafts- } \\
\text { kunde }\end{array}$ & \multirow[t]{2}{*}{70} \\
\hline Partnerin J & IP-J & $45-55$ & Werkrealschule & Fachlehrerin; Deutsch & \\
\hline Partner K & IP-K & $35-45$ & Gymnasium & $\begin{array}{l}\text { Klassenlehrer; Englisch, } \\
\text { Sport, Informatik }\end{array}$ & 45 \\
\hline Partnerin L & IP-L & $35-45$ & Realschule & $\begin{array}{l}\text { Klassenlehrerin; Deutsch, } \\
\text { Biologie }\end{array}$ & 35 \\
\hline
\end{tabular}

Tab. 1.: Übersicht der interviewten Lehrpersonen.

Zur weiteren Analyse sind die erhobenen Daten der Experteninterviews vollständig transkribiert worden, um die qualitative Inhaltsanalyse nach Mayring (2010, 601 f.) strukturiert durchführen zu können. Die Transkriptionsregeln sind in Anlehnung an Gläser und Laudel angewendet worden (Ahlrichs 2012, 110). Nach Mayring stehen die Kategorien - hier im Sinne der Einflussfaktoren aus dem UTAUT-Modell - im Zentrum der

3 Der Teil des Gesprächs mit Interviewpartnerin A wurde im weiteren Verlauf aus der Auswertung genommen. Der Grund dafür ist, dass sich im Nachgang gezeigt hat, dass IP-A ausschliesslich im Grundschulbereich unterrichtet und daher möglicherweise andere Gründe für die (Nicht-)Nutzung von Medien vorliegen im Vergleich zum Sekundarbereich. 
qualitativen Analyse, die dabei definiert, mit Ankerbeispielen beschrieben und mit klaren Regeln zur Zuordnung versehen werden müssen (Mayring 2015, 47). Nachfolgende Tabelle enthält die den Kategorien unterliegenden Kodierungsregeln und Beispiele.

\begin{tabular}{|c|c|c|}
\hline Kategorie & Kodierregel & Ankerbeispiel \\
\hline $\begin{array}{l}\text { Erwarteter } \\
\text { Mehrwert }\end{array}$ & $\begin{array}{l}\text { Wenn der Inhalt auf die didakti- } \\
\text { sche und arbeitsorganisatorische } \\
\text { Leistungssteigerung abzielt }\end{array}$ & $\begin{array}{l}\text { «Es gibt die Möglichkeit zu diffe- } \\
\text { renzieren, gerade wenn sie ihre } \\
\text { eigenen Geräte einsetzen.» } \\
\text { «Dennoch habe ich gemerkt, } \\
\text { dass die Motivation trotzdem, } \\
\text { auch wenn man es oft einsetzt, } \\
\text { höher ist.» }\end{array}$ \\
\hline $\begin{array}{l}\text { Geschätzter } \\
\text { Aufwand }\end{array}$ & $\begin{array}{l}\text { Wenn inhaltlich die Wahrneh- } \\
\text { mung zum Erwerb von Medien- } \\
\text { kompetenzen und der Aufwand } \\
\text { zur Mediennutzung erkennbar } \\
\text { ist. }\end{array}$ & $\begin{array}{l}\text { «Ich habe mich halt nicht wirk- } \\
\text { lich mit dem Gerät befasst.» } \\
\text { «Und dann scheitert es genau an } \\
\text { so Sachen, wie dem Kabel. Und } \\
\text { dann ist die Frustration ziemlich } \\
\text { schnell erreicht.» }\end{array}$ \\
\hline $\begin{array}{l}\text { Sozialer } \\
\text { Einfluss }\end{array}$ & $\begin{array}{l}\text { Wenn der Inhalt auf (schul-)ex- } \\
\text { terne Einflüsse abzielt. }\end{array}$ & $\begin{array}{l}\text { «Medienkompetenz wollen die } \\
\text { Eltern immer, dass das in der } \\
\text { Schule gemacht wird, aber das } \\
\text { kriegt man nirgends unter.» } \\
\text { «Also wenn wir, wie vor ein paar } \\
\text { Wochen, den Eltern eine kurze } \\
\text { Information zum Halbjahr geben, } \\
\text { sind die eher erschlagen von } \\
\text { unserem technischen Standard } \\
\text { hier.» }\end{array}$ \\
\hline $\begin{array}{l}\text { Unterstüt- } \\
\text { zende Be- } \\
\text { dingungen }\end{array}$ & $\begin{array}{l}\text { Wenn sich der Inhalt auf das } \\
\text { Umfeld in der Schule bezieht, } \\
\text { insbesondere auf das Kollegium, } \\
\text { die Gestaltungsfreiheit und den } \\
\text { Faktor Zeit. }\end{array}$ & $\begin{array}{l}\text { «Es waren lange Diskussionen } \\
\text { und Umfragen, bis wir zur aktuel- } \\
\text { len Medienregelung gekommen } \\
\text { sind.» } \\
\text { «Wir haben ein Intranet. Da } \\
\text { gibt es in jeder Abteilung unter- } \\
\text { schiedliche Möglichkeiten, Unter- } \\
\text { richtsmaterialien auf einem Ser- } \\
\text { ver oder einer Cloud zu teilen.» }\end{array}$ \\
\hline
\end{tabular}




\begin{tabular}{|l|l|l|}
\hline \multicolumn{1}{|c|}{ Kategorie } & \multicolumn{1}{|c|}{ Kodierregel } & \multicolumn{1}{|c|}{ Ankerbeispiel } \\
\hline $\begin{array}{l}\text { Selbstbezo- } \\
\text { gene Über- } \\
\text { zeugung/ } \\
\text { Kenntnisse } \\
\text { und Sicher- } \\
\text { heit }\end{array}$ & $\begin{array}{l}\text { Wenn das Gesagte auf die media- } \\
\text { len Kenntnisse sowie die Sicher- } \\
\text { heit im Umgang mit digitalen }\end{array}$ & $\begin{array}{l}\text { «edien schliessen lässt. } \\
\text { weniger selber wissen und vor- } \\
\text { bereiten, aber da ist schon die } \\
\text { Hürde gross.» } \\
\text { «...] lade es mir runter falls das } \\
\text { Internet wieder nicht tut und } \\
\text { habe dann eigentlich meinen } \\
\text { ganzen Unterricht auf dem Han- } \\
\text { dy drauf.» }\end{array}$ \\
\hline $\begin{array}{l}\text { Einstellung } \\
\text { zur Techno- } \\
\text { logie / Medi- } \\
\text { aler Habitus }\end{array}$ & $\begin{array}{l}\text { Wenn das Gesagte auf die per- } \\
\text { sönliche Grundeinstellung der } \\
\text { Lehrkräfte schliessen lässt. }\end{array}$ & $\begin{array}{l}\text { «Wenn du mich davon überzeu- } \\
\text { gen willst, im grossen Stile digita- } \\
\text { le Medien zu Unterrichtszwecken } \\
\text { einzusetzen, wird das vermutlich } \\
\text { schwierig sein, weil ich natürlich } \\
\text { auch mein Bild in mir trage.» }\end{array}$ \\
\hline
\end{tabular}

Tab. 2.: Kodierleitfaden und Definition der Kategorien.

Auf die Zuordnung zu den Kategorien aufbauend wurde durch drei unabhängige Kodierer eine Einordnung durchgeführt, welche zu jede Variable die Ausprägung der interviewten Personen bewerteten; beispielsweise, ob eine häufige, gelegentliche oder seltene Mediennutzung im Unterricht ableitbar ist. Für die Intercoderreliabilität (Kolb 2004, 337) der hier nominal vorliegenden Werte zur Einordnung der Ausprägungen der Interviewten in Bezug auf die UTAUT-Variablen wurde die paarweise Übereinstimmung der drei Kodierer sowie Krippendorffs Alpha bestimmt. ${ }^{4}$

Die Ergebnisse je Variable sind im Anhang zu finden. Dabei ist zu beachten, dass für die paarweise Übereinstimmung die genannten Grenzwerte durchgehend erfüllt werden, jedoch bei der Einstellung zur Technologie Krippendorffs Alpha keine zufriedenstellende Grösse annimmt. Die Abweichungen sind vorwiegend durch einen Kodierer zustande gekommen, was ggf. auf ein anderes Verständnis der Variablen im Vergleich zu den beiden anderen Kodierern hinweist. Die Einordnungen zur Technologieeinstellung müssen entsprechend kritisch betrachtet werden, sind jedoch aufgrund der hohen paarweisen Übereinstimmung weiterhin im vorliegenden Beitrag berücksichtigt und Diskrepanzen transparent dargestellt worden.

4 Zur Begründung der Verwendung der Masse für die Intercoderreliabilität und Grenzwerte: s. Anhang. 


\section{Ergebnisse - Ausprägung der subsumierten Kategorien der Interviewpartner}

Folgende Tabelle fasst die Ergebnisse der Auswertung zusammen und stellt die Ausprägungen der Antworten je Einflussfaktor dar.

\begin{tabular}{|c|c|c|c|c|c|c|c|c|c|c|c|}
\hline & $\begin{array}{l}\stackrel{m}{\vdots} \\
\cong\end{array}$ & $\begin{array}{l}\stackrel{L}{\vdots} \\
\stackrel{-}{\underline{a}}\end{array}$ & $\begin{array}{l}\underline{u} \\
\underline{\varrho}\end{array}$ & $\begin{array}{l}\stackrel{\nearrow}{\prime} \\
\cong\end{array}$ & $\stackrel{+}{\varrho}$ & $\stackrel{I}{\stackrel{I}{\prime}}$ & $\begin{array}{l}\bigcup_{1} \\
\stackrel{1}{\varrho}\end{array}$ & $\begin{array}{l}\cup \\
\stackrel{1}{\varrho}\end{array}$ & $\stackrel{0}{\underline{1}}$ & $\bar{\varrho}$ & $\stackrel{r}{\varrho}$ \\
\hline Altor & $25-$ & $45-$ & $35-$ & $35-$ & $35-$ & 25- & 55- & $25-$ & 45- & 45- & 45- \\
\hline Alter & 35 & 55 & 45 & 45 & 45 & 35 & 65 & 35 & 55 & 55 & 55 \\
\hline Geschlecht & $M$ & $M$ & $M$ & $M$ & W & W & $M$ & W & W & $M$ & W \\
\hline $\begin{array}{l}\text { Mediennut- } \\
\text { zung }\end{array}$ & & & & & & & & & & & \\
\hline $\begin{array}{l}\text { Erwarteter } \\
\text { Mehrwert }\end{array}$ & & & & & & & & & & & \\
\hline $\begin{array}{l}\text { Geschätzter } \\
\text { Aufwand }\end{array}$ & & & & & & & & & & & \\
\hline $\begin{array}{l}\text { Selbstbezo- } \\
\text { gene Über- } \\
\text { zeugung/ } \\
\text { Kenntnisse + } \\
\text { Sicherheit }\end{array}$ & & & & & & & & & & & \\
\hline $\begin{array}{l}\text { Unterstützen- } \\
\text { de Bedingun- } \\
\text { gen }\end{array}$ & & & & & & & & & & & \\
\hline $\begin{array}{l}\text { Einstellung } \\
\text { ggü. Techno- } \\
\text { logien/Media- } \\
\text { ler Habitus }\end{array}$ & & & & & & & & & & & \\
\hline $\begin{array}{l}\text { Sozialer Ein- } \\
\text { fluss }\end{array}$ & & & & & & & & & & & \\
\hline
\end{tabular}

\begin{tabular}{|c|c|c|}
\hline $\begin{array}{l}\text { Regelmässi- } \\
\text { ger/häufiger } \\
\text { Einsatz }\end{array}$ & $\begin{array}{l}\text { Situativer/ } \\
\text { gelegentli- } \\
\text { cher Einsatz }\end{array}$ & $\begin{array}{l}\text { Kein/selte- } \\
\text { ner Einsatz }\end{array}$ \\
\hline $\begin{array}{l}\text { Klarer Mehr- } \\
\text { wert des } \\
\text { Medienein- } \\
\text { satzes wird } \\
\text { gesehen }\end{array}$ & $\begin{array}{l}\text { Vereinzelte } \\
\text { Vorteile }\end{array}$ & $\begin{array}{l}\text { Kaum Mehr- } \\
\text { wert erkenn- } \\
\text { bar }\end{array}$ \\
\hline $\begin{array}{l}\text { Kein bis } \\
\text { geringer } \\
\text { zusätzlicher } \\
\text { Aufwand/Ri- } \\
\text { siken durch } \\
\text { Medienein- } \\
\text { satz }\end{array}$ & $\begin{array}{l}\text { Vereinzelter } \\
\text { Mehrauf- } \\
\text { wand im } \\
\text { Umgang }\end{array}$ & $\begin{array}{l}\text { Deutlicher } \\
\text { Mehrauf- } \\
\text { wand / hö- } \\
\text { here Risiken } \\
\text { bei Einsatz }\end{array}$ \\
\hline $\begin{array}{l}\text { Überwie- } \\
\text { gend sicher } \\
\text { im Umgang } \\
\text { und Einsatz }\end{array}$ & $\begin{array}{l}\text { Für ausge- } \\
\text { wählte Medi- } \\
\text { en, im Allge- } \\
\text { meinen eher } \\
\text { unsicher }\end{array}$ & $\begin{array}{l}\text { Kaum Kennt- } \\
\text { nisse, Unsi- } \\
\text { cherheit im } \\
\text { Umgang }\end{array}$ \\
\hline $\begin{array}{l}\text { Medienbil- } \\
\text { dung wird } \\
\text { gefördert }\end{array}$ & $\begin{array}{l}\text { Keine For- } \\
\text { derung nach } \\
\text { Medienein- } \\
\text { satz }\end{array}$ & \\
\hline $\begin{array}{l}\text { Positive } \\
\text { Grundhal- } \\
\text { tung gegen- } \\
\text { über digita- } \\
\text { len Medien }\end{array}$ & $\begin{array}{l}\text { Neutrale } \\
\text { Grundhal- } \\
\text { tung mit Pro } \\
\text { und Kontra } \\
\text { Aspekten }\end{array}$ & $\begin{array}{l}\text { Überwie- } \\
\text { gend ableh- } \\
\text { nende Hal- } \\
\text { tung gegen- } \\
\text { über neuen } \\
\text { Medien } \\
\end{array}$ \\
\hline $\begin{array}{l}\text { Externe Ein- } \\
\text { flüsse wur- } \\
\text { den durch } \\
\text { die IP nicht } \\
\text { wahrgenom- } \\
\text { men }\end{array}$ & $\begin{array}{l}\text { Medienein- } \\
\text { satz wird } \\
\text { von externen } \\
\text { Einflüssen } \\
\text { möglicher- } \\
\text { weise beein- } \\
\text { flusst }\end{array}$ & $\begin{array}{l}\text { Medienein- } \\
\text { satz von } \\
\text { externen } \\
\text { Einflüssen } \\
\text { unbeein- } \\
\text { flusst }\end{array}$ \\
\hline
\end{tabular}

Tab. 3.: Interviewkodierung nach den Ausprägungen der Interviewpersonen 
Zur Unterstützung der Mustererkennung wurden die interviewten Personen in der absteigenden Häufigkeit des Einsatzes von Medien aufgeführt, da die Mediennutzung der zu erklärende Faktor ist. Die Nutzungsabsicht selbst wird nicht erfragt, sie wird vielmehr im Kontext mit den Faktoren beobachtet, die laut Modell zur Nutzungsabsicht beitragen. Die aus den Interviews abgeleitete Einordnung in die Kategorien wurde nach positiv beeinflussenden Faktoren sortiert; beispielsweise, wenn ein klarer erwarteter Mehrwert für die Nutzungsabsicht und damit die Medienintegration aus Sicht der Interviewten gegeben war, wurde die Spalte in der Tabelle nach links gezogen, wobei die Reihenfolge nicht immer eindeutig ist.

Im Folgenden werden auffällige Konstellationen einzelner Interviewpartner betrachtet und übergreifende Aussagen aus den Interviews gefiltert. Eine Einzelfallanalyse jeder interviewten Person sprengt allerdings den Rahmen dieses Beitrags.

IP-B verhält sich, wie aus der Tabelle ersichtlich, hinsichtlich der Mediennutzung weitgehend wie vom UTAUT-Modell unterstützt und wie in den Hypothesen formuliert: Die Lehrkraft nutzt Medien regelmässig im Unterricht. Genannt werden Beamer, Lernplattformen mit interaktiven Aufgaben, Lernvideos, Nutzung von PowerPoint als Anforderung an Referate von Schülerinnen und Schüler. Die Gründe dafür sind - Kausalität gem. UTAUT-Modell unterstellend - weil

- IP-B vom Mehrwert der Medien überzeugt ist, beispielsweise werden Kollaborationen zwischen Schülerinnen und Schüler als Mehrwert genannt oder eine direkte Ergebnisrückmeldung bei der Bearbeitung von interaktiven Aufgaben.

- Weiterhin sieht IP-B den Aufwand für deren Nutzung inkl. Vorbereitung eher als gering an, beispielsweise durch die Möglichkeit der Vernetzung mit anderen Lehrpersonenüber Plattformen,

- fühlt sich kompetent und sicher bei Nutzung der Medien was unter anderem dadurch sichtbar ist, weil IP-B auch Kolleginnen und Kollegen die Nutzung der Medien erklärt,

- nimmt unterstützende Bedingungen wahr, beispielsweise durch die Möglichkeit der Einbindung digitaler Medien in Prüfungen von Schülerinnen und Schüler. 
- Die Einstellung Medien gegenüber ist neutral; es werden positive (beispielsweise «Lernen für das Leben» in Bezug auf die Nutzung bestimmter Handyfunktionen) und negative Aspekte (Videos werden nur durchgeklickt, aber nichts gelernt) wahrgenommen. ${ }^{5}$

- Weiterhin werden soziale Einflüsse von aussen in Bezug auf Mediennutzung wahrgenommen, beispielsweise wird eine gesellschaftliche Verantwortung beim Umgang mit sozialen Medien gesehen. Ob die äusseren Einflüsse die Mediennutzung im Unterricht konkret beeinflussen, ist aus dem Interview heraus nicht ableitbar.

Ein Gegenpart zu IP-B hinsichtlich der Nutzung der Medien im Unterricht ist IP-J. Auch hier ist das Muster klar:

- IP-J nutzt keine digitalen Medien,

- sieht keinen Mehrwert - beispielsweise, weil unbekannt sei, auf was man die Schülerinnen und Schüler vorbereiten solle und die Medien nicht flexibel genug seien,

- hält den Aufwand für hoch,

- fühlt sich unsicher im Umgang mit Medien (nutzt beispielsweise nie den Computerraum, empfindet es als schwierig, den CD-Player im Fach Deutsch als Zweitsprache einzusetzen),

- sieht keine unterstützenden Bedingungen oder Forderung des Medieneinsatzes durch die Schulleitung,

- hat eine überwiegend ablehnende Haltung gegenüber digitalen Medien («die Medien mögen mich nicht»),

- auch über die Familie werden digitale Medien eher negativ wahrgenommen als «Abtauchen» der Kinder in die Medien.

Nicht alle Interviewpartnerinnen und -Partner weisen ein Muster auf, das ähnlich eindeutig geprägt ist wie die von IP-B und IP-J.

Beispielsweise nutzt IP-H regelmässig Medien im Unterricht, obwohl die Lehrkraft nur vereinzelt Vorteile bei partiell hohem Aufwand sieht und im Allgemeinen eher unsicher im Umgang mit Medien ist. Als Mehrwert

5 Hinweis: Die Einstufung der Aussagen von IP-B zur Grundeinstellung zu Medien in die Kategorie "positive und negative Aspekte» wurde übereinstimmend von allen drei Ratern bewertet; ebenso die Einstufung von IP-J als «überwiegend negativ» im folgenden Absatz. 
wird beispielsweise die Möglichkeit der Unterrichtsvorbereitung über PowerPoint gesehen und die damit verbundene Visualisierung der Arbeitsaufträge für die Klasse oder die Möglichkeit der Vernetzung der Schülerinnen und Schüler untereinander. Als Aufwand bei der Nutzung werden Probleme der Dokumentenkamera genannt, was sich beispielsweise durch eine schwierige Sichtbarkeit an der Wand zeigt. Auf der anderen Seite sind unterstützende Bedingungen gegeben, beispielsweise, indem die Schule generell offen ist für neue Projekte und eine Digitalisierungsentwicklungsstelle vorhanden ist. Auch eine grundsätzlich positive Haltung gegenüber Medien ist zu beobachten, was durch Aussagen wie «Ich sehe [...] viele Chancen durch Medien an der Schule, [...] dass man das Unterrichtsfach einfach spannender [...] und anschaulicher unterrichten kann» unterstützt wird. Zudem hat IP-H Fortbildungen zur Medienintegration besucht und ein sozialer Einfluss wird teilweise durch die Forderungen von Eltern zur Medienbildung wahrgenommen.

Eine mögliche Erklärung für den häufigen Einsatz der Medien bei wenig Kenntnissen ist die geringe Bandbreite an Medien: Die Lehrkraft IP-H setzt vorwiegend PowerPoint und Dokumentenkameras ein und diese häufig. Möglicherweise beherrscht die Person diese Medien sehr gut, ist aber, ggf. aufgrund der Eigeneinschätzung, schlechte Kenntnisse zu haben, nicht bereit dazu, weitere Medien im Unterricht einzusetzen.

Ein weiteres Beispiel für ein uneindeutiges Muster ist IP-G, welcher Medien nur situativ im Unterricht einsetzt (Beamer, gelegentlich das interaktive Whiteboard, Smartphones von Schülerinnen und Schüler für Dokumentationen) obgleich eines erwarteten Mehrwerts, einer positiven Einstellung gegenüber Medien ${ }^{6}$ und nach eigenen Angaben guten Kenntnissen der Mediennutzung und vereinzelten Veranstaltungen zur Medienintegration. Der erwartete Mehrwert zeigt sich beispielsweise durch Aussagen wie

«ohne das [Internet] könnte man sich z. B. viele Unterrichtsarten gar nicht mehr vorstellen. Ich suche mal schnell einen Film zu Gewindeschneiden. [...] Jetzt habt ihr vielleicht mal ausprobieren können, jetzt

6 Die Aussagen von IP-G zur Einstellung werden von zwei der drei Rater als positiv wahrgenommen, der dritte Rater sieht eine neutrale Haltung. 
schaut ihr Mal wie es der Profi macht. Dann gehen wir nochmals auf die Feinheiten ein. Das finde ich eigentlich schon fortschrittlich im Vergleich zur Schiefertafel.»

Die Konstellation der nur gelegentlichen Nutzung trotz sonst positiv unterstützenden Aspekten wäre dann erklärbar, wenn der Aufwand und ggf. auch die unterstützenden Bedingungen und der soziale Einfluss sich stark konträr auswirken. Tatsächlich ist erkennbar, dass IP-G den Aufwand beispielsweise beim Erstellen von Präsentationen als hoch einschätzt, jedoch bei vorliegenden Unterstützungen, z. B. eine Erstellung der Präsentation durch das Kollegium, diese nutzen würde. Das ist gem. Angaben von IP-G allerdings nur in Einzelfällen so. Aufgrund mangelnder Kenntnisse wird die didaktische Einbindung nicht immer als reibungslos wahrgenommen. Weiterhin kommt bei den Kenntnissen durch, dass bei einer Ausweitung der Mediennutzung die Gefahr gesehen wird, die Schülerinnen und Schüler seien weiter als er und er als inkompetent wahrgenommen werden könnte:

«Ich habe jetzt mal eine Fortbildung besucht. Das ist so ein interaktives Männchen, wo man damit so verschiedene Blöcke dann bewegen kann. [...] Und für mich war das eher so, dass ich wahrscheinlich nicht viel weiter bin, wie die Schüler, die das Ding hingestellt bekommen und die dann intuitiv schon das Ding bewegen, weil sie aus irgendeinem Computerspiel das Ding und diese Befehle schon mal wissen. Ich kenne mich selbst nicht aus, weil ich keine Computerspiele mache».

Der soziale Einfluss wird im Interview insofern angesprochen, als dass Eltern eine Medienbildung fordern würden, aber gleichzeitig nicht wüssten, was mit Medien «anzufangen» ist.

\section{Ergebnisdiskussion}

Insgesamt zeigt das durch die Tabelle gebildete Muster, dass im Grossen und Ganzen die bisherigen Erkenntnisse zur Nutzung(sabsicht) von Medien bestätigt werden, die im Rahmen von Forschungen zum UTAUT-Modell 
gewonnen wurden, wenngleich auch nicht jeder Teil der Hypothesen für jede Person bestätigt werden kann.

Die Einzelfallbetrachtung der interviewten Personen zeigt, dass nicht alle Faktoren für die tatsächliche Nutzung relevant sind und teilweise auch nicht vollständig im Modell abgebildet werden. Insbesondere der erwartete Mehrwert und der geschätzte Aufwand scheinen für die Nutzung relevant zu sein, so dass in diesem Bereich Massnahmen angesetzt werden sollten.

Die Lösung für einen Mehreinsatz von Medien kann, wie die Interviewpartner implizit darlegen, durch gezielte Unterstützung beim Einsatz beispielsweise durch das Kollegium (IP-D, IP-E, IP-G, IP-K) oder das in einigen Interviews angesprochene Material von Schulbuchverlagen (IP-C, IP-D, IPL) und Aufzeigen der didaktischen Einbindung, die von IP-B, IP-G und IP-K angesprochen wird, gefördert werden. Der zu erwartende Aufwand wird von den Interviewten, die Medien situativ oder selten nutzen, auch häufig als erhöht und hinderlich wahrgenommen. Man müsse sich in die Medien erst «einfuchsen» (IP-D), würde diese eher nutzen, wenn schon ein auf den Unterricht abgestimmter Pool von Materialien vorliegen (IP-I) oder eine sukzessive Heranführung stattfinden würde (IP-B). Mit solchen Angeboten werden Einstiegshürden niedriger gelegt und die mangelnden Kenntnisse und damit Gefahr, sich vor den Schülerinnen und Schüler blosszustellen (IP-D, IP-G) gesenkt.

Weitere Erkenntnisse lassen sich durch Interviewpassagen gewinnen, welche die Bedeutung der definierten Kategorien tw. ergänzen: Ein Aspekt lässt sich in der technischen Ausstattung der Schulen finden. Dieser Bereich war zwar nicht direkt Gegenstand der Untersuchung, jedoch lassen sich aus den Bewertungen der Lehrenden zu den technisch-organisatorischen Rahmenbedingungen weitere Hemmnisse schliessen. So wird trotz der in den Schulen der Interviewten grundsätzlich vorhandenen Medienausstattung von mehreren interviewten Personen die dahinterliegende Infrastruktur (kein W-LAN oder ausgefallene Server wurden durch sechs von elf Personen moniert) oder auch unterschiedlich ausgestattete Klassenräume bemängelt, was die Übertragung eines digitalen Unterrichtskonzepts in unterschiedlichen Klassen zumindest erschwert. 
Ein wichtiger und häufig erwähnter Faktor ist jener der Ausfallsicherheit sowie der des technischen Supports. Digitalen Medien wird im Allgemeinen eine gewisse Zuverlässigkeit abgesprochen, was bei herkömmlichen Medien wie der Schiefertafel nicht der Fall ist. Selbst Befürwortern neuer Medien sind die Risiken des Ausfalls technischer Systeme bewusst, worauf sie sich unterschiedlich vorbereiten, indem beispielsweise alle unterrichtsrelevanten Inhalte bereits zu Hause heruntergeladen oder traditionelle Medien im Notfall eingesetzt werden.

Die Ergebnisse zu den Faktoren des Einsatzes digitaler Medien durch Lehrende sind dahingehend kritisch zu beurteilen, dass diese keine Aussagekraft über die Qualität des Einsatzes zulassen - ein Aspekt, der von Drossel et al. $(2019,234)$ in Bezug auf die Art der eingesetzten Medien aufgegriffen wird. Auch die Bandbreite an genutzten Medien wird durch das Modell nicht angesprochen (s. dazu Drossel et al. 2019, 217 ff.). Dabei kann auch der häufigere Einsatz von einzelnen Medien zu einer vermehrten Nutzung führen, wenn der Einsatz digitaler Medien im Unterricht im Allgemeinen beabsichtigt wird. Von mehreren Interviewpartnern werden nur wenige und eher einfach zu bedienende Medien (z. B. Beamer, Dokumentenkamera) genutzt, diese dafür aber häufig und gezielt. Die Untersuchung der Qualität und Bandbreite des Einsatzes zählt nicht zum Anspruch dieser Arbeit, könnte jedoch in einer weiteren Untersuchung berücksichtigt werden. Weiterhin wurden die Schülerinnen und Schüler der interviewten Lehrpersonen und deren Sichtweise z. B. auf die Lernförderlichkeit des $\mathrm{Me}$ dieneinsatzes nicht berücksichtigt, was neue Erkenntnisse mit sich bringen würde.

Auch ist kritisch anzumerken, dass die Stichprobe möglicherweise verzerrt ist, da die Interviewten alle in Schulen in Baden-Württemberg lehren und durch die Rahmenbedingungen der dortigen Schulpolitik beeinflusst sind.

Die Durchführung einer qualitativen Untersuchung hat weiterhin nicht den Anspruch, eine allgemeingültige Aussage zu treffen und das Ergebnis deduktiv auf alle Lehrenden in Deutschland zu übertragen. Vielmehr sollen hierbei Einzelfälle veranschaulicht und anhand der Einzelperson dargestellt werden, aus welchen Gründen es zu einer (Nicht-)Nutzung von Medien kommt. 
Anzumerken ist weiterhin, dass die Interviews vor der Corona-Pandemie geführt wurden und damit zu Zeiten, in denen zumindest kein grösserer äusserer Zwang zum Medieneinsatz in der breiten Öffentlichkeit diskutiert wurde. Die Sichtweise der Interviewten «vor und nach Corona» könnte interessante Erkenntnisse bringen, ob und wie sich der Medieneinsatz gewandelt hat.

Es ist anzunehmen, dass die Schule den Anforderungen einer mediatisierten Gesellschaft nur dann gerecht werden kann, wenn Lehrpersonen über ein ausreichendes Mass an Medienkompetenzen verfügen. Dabei zählen sowohl das kritische Hinterfragen als auch der selbstsichere Umgang mit digitalen Endgeräten und das Zurechtfinden in der digitalen Welt. Grundvoraussetzung dafür ist eine funktionierende Infrastruktur. Unterstützt werden können die Lehrpersonen über niederschwellige Angebote wie beispielsweise eine konkrete Unterstützung im Kollegium oder anderer Vernetzung von Lehrenden sowie einen Pool an auf den Unterricht abgestimmten Unterlagen. Lehrpersonen sind Vorbilder für ihre Schülerinnen und Schüler, welche auf ein Leben in einer Mediengesellschaft vorbereitet werden sollen. Daher ist es unabdingbar, dass sich die Lehrperson mit dieser Thematik befasst und zumindest in Teilen einen vorbildlichen Umgang mit digitalen Medien vorlebt.

\section{Literatur}

Ahlrichs, Rolf. 2012. Zwischen sozialer Verantwortung und ökonomischer Vernunft: Unternehmensethische Impulse für die Sozialwirtschaft. Wiesbaden: VS Verlag für Sozialwissenschaften. https://doi.org/10.1007/978-3-531-94355-8.

Ameri, Arefeh, Reza Khajouei, Alieh Ameri, und Yunes Jahani. 2020. «Acceptance of a Mobile-Based Educational Application (LabSafety) By Pharmacy Students: An Application of the UTAUT2 Model». Education And Information Technologies 25 (1): 419-35. https://doi.org/10.1007/s10639-019-09965-5.

Atteslander, Peter, und Jürgen Cromm. 2010. Methoden der empirischen Sozialforschung. 13., neu bearb. und erw. Aufl. ESV basics. Berlin: Erich Schmidt.

Bach, Alexandra. 2016. «Nutzung von digitalen Medien an berufsbildenden Schulen: Notwendigkeit, Rahmenbedingungen, Akzeptanz und Wirkungen». In Jahrbuch der berufs- und wirtschaftspädagogischen Forschung 2016, herausgegeben von Jürgen Seifried, Susan Seeber, und Birgit Ziegler, 107-23. Schriftenreihe der Sektion Berufs- und Wirtschaftspädagogik der Deutschen Gesellschaft für Erziehungswissenschaft (DGfE) v. 2015/16. Leverkusen-Opladen: Barbara Budrich. https://doi.org/10.3224/84740588. 
Biermann, Ralf. 2009. Der mediale Habitus von Lehramtsstudierenden: Eine quantitative Studie zum Medienhandeln angehender Lehrpersonen. Wiesbaden: VS Verlag für Sozialwissenschaften / GWV Fachverlage GmbH. https://doi. org/10.1007/978-3-531-91501-2.

Birch, Anthony, und Valerie Irvine. 2009. «Preservice Teachers' Acceptance of ICT Integration in the Classroom: Applying the UTAUT Model». Educational Media International 46 (4): 295-315. https://doi.org/10.1080/09523980903387506.

Brandhofer, Gerhard. 2015. «Die Kompetenzen der Lehrenden an Schulen im Umgang mit digitalen Medien und die Wechselwirkungen zwischen Lehrtheorien und mediendidaktischem Handeln: Dissertation». http://nbn-resolving.de/ urn:nbn:de:bsz:14-qucosa-190208.

Breiter, Andreas, Björn Eric Stolpmann, und Stefan Welling. 2010. «Medienkompetenz in der Schule - Integration von Medien in den weiterführenden Schulen in Nordrhein-Westfalen: Kurzfassung der Untersuchung». Schriftenreihe Medienforschung der Landesanstalt für Medien Nordrhein-Westfalen. Berlin: Vistas. https://www.medienanstalt-nrw.de/fileadmin/lfm-nrw/Forschung/ LfM-Band-64.pdf.

Drossel, Kerstin, Birgit Eickelmann, Heike Schaumburg, und Amelie Labusch. 2019. «Nutzung Digitaler Medien Und Prädiktoren Aus Der Perspektive Der Lehrerinnen Und Lehrer Im Internationalen Vergleich». In ICILS 2018 \#Deutschland: Computer- Und Informationsbezogene Kompetenzen Von Schülerinnen Und Schülern Im Zweiten Internationalen Vergleich Und Kompetenzen Im Bereich Computational Thinking, herausgegeben von Birgit Eickelmann, Wilfried Bos, und Julia Gerick, 205-40. Münster: Waxmann. http://nbn-resolving. org/urn:nbn:de:0111-pedocs-183253.

Eder, Alexandra. 2015. «Akzeptanz von Bildungstechnologien in der gewerblichtechnischen Berufsbildung vor dem Hintergrund von Industrie 4.0». Journal of Technical Education (JOTED) 3 (2). https://www.journal-of-technical-education.de/index.php/joted/article/view/57.

Eickelmann, Birgit, Wilfried Bos, Julia Gerick, Frank Goldhammer, Heike Schaumburg, Knut Schwippert, Martin Senkbeil, und Jan Vahrenhold. 2019. «ICILS 2018 \#Deutschland. Computer- Und Informationsbezogene Kompetenzen Von Schülerinnen Und Schülern Im Zweiten Internationalen Vergleich Und Kompetenzen Im Bereich Computational Thinking». Münster, New York: Waxmann. http://nbn-resolving.org/urn:nbn:de:0111-pedocs-181664.

Feierabend, Sabine, Theresa Plankenhorn, und Thomas Rathgeb. 2017. «JIM 2017. Jugend, Information, (Multi-) Media. Basisstudie zum Medienumgang 12- bis 19-Jähriger in Deutschland». Herausgegeben von Medienpädagogischer Forschungsverbund Südwest. Medienpädagogischer Forschungsverbund Südwest. https://www.mpfs.de/fileadmin/files/Studien/JIM/2017/JIM_2017.pdf.

Garone, Anja, Bram Pynoo, Jo Tondeur, Celine Cocquyt, Silke Vanslambrouck, Bram Bruggeman, und Katrien Struyven. 2019. "Clustering University Teaching Staff Through UTAUT: Implications for the Acceptance of a New Learning Management System». British Journal of Educational Technology 50 (5): 2466-83. https://doi.org/10.1111/bjet.12867. 
Grasmück, Edith, Gerhard Büttner, und Regina Vollmeyer. 2010. «Selbstreguliertes Lernen und E-Learning in der Lehrerfortbildung. Entwicklung und Evaluation einer Fortbildungsmassnahme». In Lehrerinnen und Lehrer lernen: Konzepte und Befunde zur Lehrerfortbildung, herausgegeben von Florian H. Müller, Astrid Eichenberger, Manfred Lüders, und Johannes Mayr, 261-78. Münster, New York, München, Berlin: Waxmann.

Gysbers, Andre. 2008. «Lehrer - Medien - Kompetenz: Eine empirische Untersuchung zur medienpädagogischen Kompetenz und Performanz niedersächsischer Lehrkräfte». Schriftenreihe der NLM Bd. 22. Berlin: Vistas.

Hoffmann, Dagmar, und Lothar Mikos. 2010. «Warum dieses Buch? Einige einführende Anmerkungen». In Mediensozialisationstheorien, herausgegeben von Dagmar Hoffmann und Lothar Mikos, 7-10. Wiesbaden: VS Verlag für Sozialwissenschaften. https://doi.org/10.1007/978-3-531-92249-2_1.

Knaus, Thomas, und Katrin Valentin. 2016. «Video-Tutorials in der Hochschullehre - Hürden, Widerstände und Potentiale». In Wi(e)derstände. Digitaler Wandel in Bildungseinrichtungen, herausgegeben von Thomas Engels und Knaus, Olga, 151-181. München: kopaed.

Kolb, Steffen. 2004. «Verlässlichkeit von Inhaltsanalysedaten. Reliabilitätstest, Errechnen und Interpretieren von Reliabilitätskoeffizienten für mehr als zwei Codierer». Medien \& Kommunikationswissenschaft 52 (3): 335-54. https://doi. org/10.5771/1615-634x-2004-3-335.

Kommer, Sven. 2016. «Buch statt Tablet-PC: Warum die digitalen Medien nicht in die Schule kommen - der Faktor LehrerIn». In Wi(e)derstände. Digitaler Wandel in Bildungseinrichtungen herausgegeben von Thomas Engels und Knaus, Olga, 35-68. München: kopaed.

Kommer, Sven, und Ralf Biermann. 2012. «Der mediale Habitus von (angehenden) LehrerInnen. Medienbezogene Dispositionen und Medienhandeln von Lehramtsstudierenden». In Jahrbuch Medienpädagogik 9, herausgegeben von Renate Schulz-Zander, Birgit Eickelmann, Heinz Moser, Horst Niesyto, und Petra Grell, 81-108. Wiesbaden: VS Verlag für Sozialwissenschaften. https:// doi.org/10.1007/978-3-531-94219-3_5.

Krippendorff, Klaus. 1980. «Content Analysis: An Introduction to Its Methodology». 1. print. The Sage commtext series 5. Beverly Hills, Calif. SAGE Publ.

Krippendorff, Klaus, und Mary Angela Bock. 2009. The Content Analysis Reader. Los Angeles: Sage.

Lermen, Markus. 2008. Digitale Medien in der Lehrerbildung: Rahmenbedingungen, Einflussfaktoren und Integrationsvorschläge aus (medien-)pädagogischer Sicht. 1. Aufl. Grundlagen der Berufs- und Erwachsenenbildung Bd. 57. Baltmannsweiler: Schneider-Verl. Hohengehren.

Lauf, Edmund. 2001. «'.96 nach Holsti' Zur Reliabilität von Inhaltsanalysen und deren Darstellung in kommunikationswissenschaftlichen Fachzeitschriften». Publizistik 46 (1): 57-68. https://doi.org/10.1007/s11616-001-0004-7. 
Lin, Peng-Chun, Lu Hsin-Ke, und Shang-Chia Liu. 2013. «Toward an Education Behavioral Intention Model for E-Learning Systems: An Extension of UTAUT». Journal of Theoretical and Applied Information Technology 47(3): 1120-27. http:// www.jatit.org/volumes/Vol47No3/37Vol47No3.pdf.

Mayring, Philipp. 2010. «Qualitative Inhaltsanalyse». In Handbuch qualitative Forschung in der Psychologie, herausgegeben von Günter Mey und Katja Mruck. 1. Auflage, 601-13. Wiesbaden: VS Verlag für Sozialwissenschaften. https://doi. org/10.1007/978-3-531-92052-8_42.

Mayring, Philipp. 2015. Qualitative Inhaltsanalyse: Grundlagen und Techniken. 12. Aufl. Beltz Pädagogik. Weinheim, Bergstr: Beltz, J.

Meuser, Michael, und Ulrike Nagel. 1991. «ExpertInneninterviews - vielfach erprobt, wenig bedacht: ein Beitrag zur qualitativen Methodendiskussion». In Qualitativ-empirische Sozialforschung: Konzepte, Methoden, Analysen, herausgegeben von Detlef Garz, und Klaus Kraimer, 441-71. Opladen: Westdeutscher Verlag. https://doi.org/10.1007/978-3-322-97024-4.

Nistor, Nicolae, Maximilian Wagner, und Jan Oliver Heymann. 2012. «Prädiktoren und Moderatoren der Akzeptanz von Bildungstechnologien: Die Unified Theory of Acceptance and Use of Technology auf dem Prüfstand». Empirische Pädagogik 26 (3): 343-71.

Perienen, Appavoo. 2020. «Frameworks for ICT Integration in Mathematics Education - a Teacher's Perspective». Eurasia Journal of Mathematics, Science and Technology Education 16 (6). https://doi.org/10.29333/ejmste/7803.

Prasse, Doreen. 2012. Bedingungen Innovativen Handelns in Schulen: Funktion Und Interaktion Von Innovationsbereitschaft, Innovationsklima und Akteursnetzwerken Am Beispiel Der IKT-Integration an Schulen. Empirische Erziehungswissenschaft 38. Münster: Waxmann.

Raupp, Juliana, und Jens Vogelgesang. 2009. Medienresonanzanalyse: Eine Einführung in Theorie und Praxis. Lehrbuch. Wiesbaden: VS Verlag für Sozialwissenschaften | GWV Fachverlage GmbH. https://doi.org/10.1007/978-3-531-91605-7.

Schiefner-Rohs, Mandy, Claudia Gomez Tutor, und Christine Menzer, Hrsg. 2015. Lehrer.Bildung.Medien: Herausforderungen für die Entwicklung und Gestaltung von Schule. Grundlagen der Berufs- und Erwachsenenbildung Band 82. Baltmannsweiler: Schneider Verlag Hohengehren GmbH.

Schulz-Zander, Renate, und Birgit Eickelmann. 2008. «Qualitative Forschung in der Medienpädagogik: Zur Erfassung von Schulentwicklungsprozessen im Bereich digitaler Medien Methodologische Konzeption einer Fallstudienuntersuchung als Folgeuntersuchung zur deutschen IEA-Studie SITES M2». MedienPädagogik. Zeitschrift für Theorie und Praxis der Medienbildung (14): 1-22. https://doi.org/10.21240/mpaed/14/2008.01.31.X.

Šumak, Boštjan, und Andrej Šorgo. 2016. «The Acceptance and Use of Interactive Whiteboards Among Teachers: Differences in UTAUT Determinants Between Pre- and Post-Adopters». Computers in Human Behavior (64): 602-20. https:// doi.org/10.1016/j.chb.2016.07.037. 
Tappe, Eik-Henning. 2019. "Prädiktoren der Intention zum didaktischen Einsatz von digitalen Medien im Unterricht - Überführung der Unified Theory of Acceptance and Use of Technology (UTAUT) in ein schulisches Untersuchungssetting». In Projekt - Theorie - Methode: Spektrum Medienpädagogischer Forschung. Vol. 3, herausgegeben von Thomas Knaus, 999-1027. München: kopaed. https://doi.org/10.25526/fw-mp.35.

Venkatesh, Viswanath, Michael G. Morris, Gordon Davis, und Fred Davis. 2003. «User Acceptance of Information Technology: Toward a Unified View.» MIS Quarterly 27 (3): 425. https://doi.org/10.2307/30036540.

Venkatesh, Viswanath, James Y. L. Thong, und Xin Xu. 2012. "Consumer acceptance and use of information technology: extending the unified theory of acceptance and use of technology». MIS Quarterly (36): 157-78. https://doi. org/10.2307/41410412.

Vogl, Susanne. 2014. «Gruppendiskussion». In Handbuch Methoden Der Empirischen Sozialforschung, herausgegeben von Nina Baur, und Jörg Blasius, 58186. Wiesbaden: Springer VS. https://doi.org/10.1007/978-3-658-21308-4.

Waffner, Bettina. 2020. «Unterrichtspraktiken, Erfahrungen Und Einstellungen Von Lehrpersonen Zu Digitalen Medien in Der Schule. In Bildung Im Digitalen Wandel: Die Bedeutung Für Das Pädagogische Personal Und Für Die Aus- Und Fortbildung. Vol. 1, herausgegeben von Annika Wilmers, Carolin Anda, Carolin Keller, und Marc Rittberger, 57-102. Münster: Waxmann. https://doi. org/10.31244/9783830991991.03.

Williams, Michael D., Nripendra P. Rana, und Yogesh K. Dwivedi. 2015. «The Unified Theory of Acceptance and Use of Technology (UTAUT): A Literature Review». Journal of Enterprise Information Management 28 (3): 443-88. https://doi. org/10.1108/JEIM-09-2014-0088.

Yin, Robert K. 2003. Case Study Research: Design and Methods. 3. ed. Applied social research methods series 5. Thousand Oaks, CA: Sage. 


\section{Anhang 1:}

\begin{tabular}{|l|c|c|}
\hline \multirow{2}{*}{} & \multicolumn{2}{|c|}{ Interraterreliabilität } \\
\cline { 2 - 3 } & $\begin{array}{c}\text { Paarweise Übereinstim- } \\
\text { mung (Durchschnitt) }\end{array}$ & Krippendorffs Alpha \\
\hline Mediennutzung & $100,00 \%$ & 1,00 \\
\hline Erwarteter Mehrwert & $87,88 \%$ & 0,81 \\
\hline Geschätzter Aufwand & $87,88 \%$ & 0,82 \\
\hline $\begin{array}{l}\text { Selbstbezogene Über- } \\
\text { zeugung/Kenntnisse + } \\
\text { Sicherheit }\end{array}$ & $100,00 \%$ & 1,00 \\
\hline $\begin{array}{l}\text { Unterstützende Bedin- } \\
\text { gungen }\end{array}$ & $93,94 \%$ & 0,88 \\
\hline $\begin{array}{l}\text { Einstellung gegenüber } \\
\text { Technologien / Medialer } \\
\text { Habitus }\end{array}$ & $81,82 \%$ & 0,62 \\
\hline Sozialer Einfluss & $93,94 \%$ & 0,89 \\
\hline
\end{tabular}

Krippensdorffs Alpha (Krippendorff 1980, berechnet gem. Krippendorff und Bock 2009, 4) wurde als Messgrösse gewählt, da es sich für mehr als zwei Coder anwenden lässt, zufällige Übereinstimmungen berücksichtigt (Lauf 2001, 60) und es sich laut Kolb $(2004,340)$ bei Codierungen um Schätzungen handelt und somit ein Schätzmass als angemessen betrachtet wird. In der Literatur werden Werte von $\geq 0.8$ als akzeptabel bezeichnet (s. Raupp und Vogelgesang 2009, xxi), Lauf 2001,60 - in der letztgenannten Literatur werden Werte für Krippendorffs Alpha auch von $\geq 0.75$ als akzeptabel bezeichnet). 


\section{Anhang 2: Interviewleitfaden}

Die zu deckenden Informationsbedarfe bilden die Grundlage für den Interviewleitfaden, welcher aus den subsumierten Detailfragen zu jedem Informationsbedarf besteht. Dabei sind diese aus dem UTAUT-Modell abgeleitet worden, wie es von Tappe $(2019,1018)$ bereits auf den Bildungskontext übertragen wurde. Der Kategorie Il zielt auf die Leistungserwartung ab, I2 thematisiert die Aufwandserwartung, I3 die selbstbezogene Überzeugung, I4 die begünstigenden Bedingungen, I5 die Einstellung zur Technologienutzung sowie die damit verbundenen Befürchtungen und I6 den sozialen Einfluss.

\begin{tabular}{|c|c|c|c|}
\hline ID-I & Informationsbedarf & ID-D & Detailfrage \\
\hline $\mid 1-1$ & $\begin{array}{l}\text { Wie nehmen Lehrende den Ein- } \\
\text { satz digitaler Medien in Bezug } \\
\text { auf die Lernförderlichkeit wahr? }\end{array}$ & D1-1 & $\begin{array}{l}\text { Welche Erfahrungen haben Sie } \\
\text { beim Einsatz digitaler Medien } \\
\text { zum Lernverhalten der Schüler- } \\
\text { innen und Schüler gemacht? }\end{array}$ \\
\hline $\mid 1-2$ & $\begin{array}{l}\text { Wie werden digitale Medien in } \\
\text { Bezug auf pädagogische Lehr- } \\
\text { konzepte eingeschätzt? }\end{array}$ & D1-2 & $\begin{array}{l}\text { Wie schätzen Sie neue Medien } \\
\text { in Bezug auf pädagogische Mög- } \\
\text { lichkeiten ein? }\end{array}$ \\
\hline $\mid 1-3$ & $\begin{array}{l}\text { Wie stehen Lehrende zum Ein- } \\
\text { satz neuer Medien im Unterricht? }\end{array}$ & D1-3 & $\begin{array}{l}\text { Welche Rolle spielen digitale } \\
\text { Medien im Schulalltag? }\end{array}$ \\
\hline $\mid 1-4$ & $\begin{array}{l}\text { Empfinden Lehrende neue Me- } \\
\text { dien als weniger passend für die } \\
\text { Bildung im Vergleich zu traditio- } \\
\text { nellen Medien? }\end{array}$ & D1-4 & $\begin{array}{l}\text { Wie schätzen Sie neue Medien } \\
\text { im Vergleich zu herkömmlichen } \\
\text { Medien für den Unterricht ein? }\end{array}$ \\
\hline $\mid 1-5$ & $\begin{array}{l}\text { Wie schätzen Lehrende die Medi- } \\
\text { ennutzung der Schulkinder ein? }\end{array}$ & D1-5 & $\begin{array}{l}\text { Welche Medien werden nach } \\
\text { Ihrer Einschätzung von den } \\
\text { Schülerinnen und Schüler bevor- } \\
\text { zugt genutzt und warum? }\end{array}$ \\
\hline $\mid 1-6$ & $\begin{array}{l}\text { Wie stehen Lehrpersonen digi- } \\
\text { talen Medien allgemein gegen- } \\
\text { über? }\end{array}$ & D1-6 & $\begin{array}{l}\text { Welche Eigenschaften würden } \\
\text { Sie digitalen Medien spontan } \\
\text { zurechnen? }\end{array}$ \\
\hline $\mid 1-7$ & $\begin{array}{l}\text { Wird Schule als Schutzraum für } \\
\text { Schülerinnen und Schüler vor } \\
\text { digitalen Medien angesehen? }\end{array}$ & D1-7 & $\begin{array}{l}\text { Wie stehen Sie der Mediennut- } \\
\text { zung der Schülerinnen und Schü- } \\
\text { ler in der Schule gegenüber? }\end{array}$ \\
\hline $\mid 1-8$ & $\begin{array}{l}\text { Empfinden Lehrende den Unter- } \\
\text { richtsinhalt durch neue Medien } \\
\text { in den Hintergrund gerückt? }\end{array}$ & D1-8 & $\begin{array}{l}\text { Was wollen Sie erreichen, wenn } \\
\text { Sie digitale Medien einsetzen? }\end{array}$ \\
\hline
\end{tabular}




\begin{tabular}{|c|c|c|c|}
\hline ID-I & Informationsbedarf & ID-D & Detailfrage \\
\hline $12-1$ & $\begin{array}{l}\text { Wie wird der Aufwand zur Erler- } \\
\text { nung von Medienkompetenzen } \\
\text { wahrgenommen? }\end{array}$ & D2-1 & $\begin{array}{l}\text { Wenn Sie in } 4 \text { Wochen nur noch } \\
\text { digitale Medien im Unterricht } \\
\text { einsetzen dürften, wie würden } \\
\text { Sie vorgehen? }\end{array}$ \\
\hline $12-2$ & $\begin{array}{l}\text { Wie nehmen Lehrende mit einem } \\
\text { geübten Medienumgang den } \\
\text { Medieneinsatz wahr? }\end{array}$ & D2-2 & $\begin{array}{l}\text { Nutzen Sie im Unterricht regel- } \\
\text { mässig digitale Medien? Und } \\
\text { wenn ja, wie gehen sie hier vor? }\end{array}$ \\
\hline $12-3$ & $\begin{array}{l}\text { Wie wirkt sich die Medieninte- } \\
\text { gration im Unterricht auf die } \\
\text { wahrgenommene Belastung und } \\
\text { Zufriedenheit des Medieneinsat- } \\
\text { zes aus? }\end{array}$ & D2-3 & $\begin{array}{l}\text { Wie empfinden Sie das Unter- } \\
\text { richtsgeschehen durch den } \\
\text { Einsatz digitaler Medien im } \\
\text { Vergleich zu herkömmlichen } \\
\text { Medien? }\end{array}$ \\
\hline $12-4$ & $\begin{array}{l}\text { Inwiefern werden möglichen } \\
\text { Risiken im Vergleich potentiellen } \\
\text { Chancen eingeschätzt? }\end{array}$ & D2-4 & $\begin{array}{l}\text { Wie sehen Sie die Risiken im Ver- } \\
\text { gleich zu den Chancen durch den } \\
\text { Medieneinsatz im Unterricht? }\end{array}$ \\
\hline $12-5$ & $\begin{array}{l}\text { Inwiefern führt das Chancen-Ri- } \\
\text { siko Verhältnis zur Meidung des } \\
\text { Einsatzes digitaler Medien? }\end{array}$ & D2-5 & $\begin{array}{l}\text { Inwiefern führt das Chancen-Ri- } \\
\text { siko Verhältnis zur Meidung des } \\
\text { Einsatzes digitaler Medien? }\end{array}$ \\
\hline $13-1$ & $\begin{array}{l}\text { Wie war das Medienverhalten in } \\
\text { der eigenen Kindheit geprägt? }\end{array}$ & D3-2 & $\begin{array}{l}\text { Welche aufkommenden Medien } \\
\text { haben Sie in Ihrer Kindheit kon- } \\
\text { sumiert? }\end{array}$ \\
\hline $13-2$ & $\begin{array}{l}\text { Wie ist die grundlegende Einstel- } \\
\text { lung / Haltung gegenüber neuen } \\
\text { Medien? }\end{array}$ & D3-2 & $\begin{array}{l}\text { Wie stehen Sie zum Einsatz neu- } \\
\text { er Medien? }\end{array}$ \\
\hline $13-3$ & $\begin{array}{l}\text { Inwieweit werden neue Medien } \\
\text { und deren Einfluss reflektiert } \\
\text { und hinterfragt? }\end{array}$ & D3-3 & $\begin{array}{l}\text { Sehen Sie in digitalen Medien } \\
\text { eher Vorteile oder Nachteile für } \\
\text { das Unterrichtsgeschehen und } \\
\text { warum? }\end{array}$ \\
\hline $13-4$ & $\begin{array}{l}\text { Welche Unterrichtsformen nut- } \\
\text { zen Lehrende in Ihrem Unter- } \\
\text { richt? }\end{array}$ & D3-4 & $\begin{array}{l}\text { Wie würden Sie Ihren täglichen } \\
\text { Unterricht beschreiben? }\end{array}$ \\
\hline $13-5$ & $\begin{array}{l}\text { Wie stehen Lehrende einer sich } \\
\text { ändernden Lehrsituation durch } \\
\text { neue Medien gegenüber? / Ha- } \\
\text { ben sie ihre Lehrformen in letzter } \\
\text { Zeit angepasst oder geändert? }\end{array}$ & D3-5 & $\begin{array}{l}\text { Beschreiben Sie bitte, wie der } \\
\text { Medieneinsatz in Ihrer Schule } \\
\text { rück-blickend in den vergange- } \\
\text { nen Jahren war? }\end{array}$ \\
\hline $14-1$ & $\begin{array}{l}\text { Wie empfinden Lehrende die } \\
\text { Gestaltungsfreiheit ihres Unter- } \\
\text { richts (mit digitalen Medien)? }\end{array}$ & D4-1 & $\begin{array}{l}\text { Welche Möglichkeiten zur freien } \\
\text { Unterrichtsvorbereitung haben } \\
\text { Sie? }\end{array}$ \\
\hline
\end{tabular}




\begin{tabular}{|c|c|c|c|}
\hline ID-I & Informationsbedarf & ID-D & Detailfrage \\
\hline $14-2$ & $\begin{array}{l}\text { Fordert die Schulorganisation } \\
\text { Fähigkeiten der Medienkompe- } \\
\text { tenz? }\end{array}$ & D4-2 & $\begin{array}{l}\text { Gibt es an Ihrer Schule Regeln } \\
\text { oder Forderungen zum Einsatz } \\
\text { digitaler Medien? }\end{array}$ \\
\hline $14-3$ & $\begin{array}{l}\text { Fühlen sich Lehrende von For- } \\
\text { derungen der Schulorganisation } \\
\text { unter Druck gesetzt? }\end{array}$ & D4-3 & $\begin{array}{l}\text { Wie wirken sich die Forderungen } \\
\text { auf das Kollegium aus? }\end{array}$ \\
\hline $14-4$ & $\begin{array}{l}\text { Fördert die Schulleitung die } \\
\text { Kommunikation im Kollegium } \\
\text { und wie? }\end{array}$ & D4-4 & $\begin{array}{l}\text { Was unternimmt die Schullei- } \\
\text { tung zum Austausch der Lehren- } \\
\text { den untereinander? }\end{array}$ \\
\hline $14-5$ & $\begin{array}{l}\text { Welche Meinungen herrschen im } \\
\text { Kollegium hinsichtlich der Medi- } \\
\text { enbildung? }\end{array}$ & D4-5 & $\begin{array}{l}\text { Welche Debatten finden im Kol- } \\
\text { legium hinsichtlich der Medien- } \\
\text { nutzung statt? }\end{array}$ \\
\hline $14-6$ & $\begin{array}{l}\text { Wie kommen Lehrende mit der } \\
\text { verfügbaren Zeit zur Unterrichts- } \\
\text { vorbereitung aus? }\end{array}$ & D4-6 & $\begin{array}{l}\text { Wie kommen Sie mit der verfüg- } \\
\text { baren Zeit zur Unterrichtsvorbe- } \\
\text { reitung aus? }\end{array}$ \\
\hline $15-1$ & $\begin{array}{l}\text { Wie empfinden Lehrende die } \\
\text { technische Entwicklung? }\end{array}$ & D5-1 & $\begin{array}{l}\text { Wie nehmen Sie die technische } \\
\text { Entwicklung wahr? }\end{array}$ \\
\hline $15-2$ & $\begin{array}{l}\text { Welche Einstellung haben sie } \\
\text { gegenüber dem technischen } \\
\text { Wandel? }\end{array}$ & D5-2 & $\begin{array}{l}\text { Welche Einstellung haben Sie } \\
\text { gegenüber dem technischen } \\
\text { Wandel? }\end{array}$ \\
\hline $15-3$ & $\begin{array}{l}\text { Wie werden die eigenen Bedien- } \\
\text { Kompetenzen eingeschätzt? }\end{array}$ & D5-3 & $\begin{array}{l}\text { Wie schätzen Sie Ihren eigenen } \\
\text { Umgang mit neuen Medien ein? }\end{array}$ \\
\hline $15-4$ & $\begin{array}{l}\text { Wie sicher fühlen sie sich beim } \\
\text { Einsatz neuer Medien im Unter- } \\
\text { richt und warum? }\end{array}$ & D5-4 & $\begin{array}{l}\text { Wie empfinden Sie das Unter- } \\
\text { richtsgeschehen durch den Ein- } \\
\text { satz digitaler Medien? }\end{array}$ \\
\hline $15-5$ & $\begin{array}{l}\text { Wie bereiten sich Lehrende auf } \\
\text { den Unterricht vor? }\end{array}$ & D5-5 & $\begin{array}{l}\text { Wie bereiten Sie Ihre Materialien } \\
\text { für den Unterricht vor? }\end{array}$ \\
\hline $16-1$ & $\begin{array}{l}\text { Nehmen Lehrende einen sozia- } \\
\text { len Druck der Eltern wahr? }\end{array}$ & D6-1 & $\begin{array}{l}\text { Welche Äusserungen der Eltern } \\
\text { zu digitalen Medien sind Ihnen } \\
\text { in Erinnerung geblieben und } \\
\text { warum? }\end{array}$ \\
\hline $16-1$ & $\begin{array}{l}\text { Wie reagieren sie auf mögliche } \\
\text { Eindrücke der sozialen Einfluss- } \\
\text { nahme? }\end{array}$ & D6-2 & $\begin{array}{l}\text { Welche Konsequenzen haben } \\
\text { Sie für sich aus den Äusserungen } \\
\text { gezogen? }\end{array}$ \\
\hline
\end{tabular}


Die in der nachfolgenden Tabelle dargestellten Leitfragen des Interviews sind aus den dazugehörigen Detailfragen subsumiert worden und bilden das Grundgerüst der geführten Interviews.

\begin{tabular}{|c|c|c|c|}
\hline Leitfragen & ID-F & Detailfragen & ID-D \\
\hline \multirow[t]{3}{*}{$\begin{array}{l}\text { Wie würden Sie Ihren täglichen } \\
\text { Unterricht beschreiben? }\end{array}$} & \multirow[t]{3}{*}{ F1 } & $\begin{array}{l}\text { Welche Rolle spielen digitale } \\
\text { Medien im Schulalltag? }\end{array}$ & D1-3 \\
\hline & & $\begin{array}{l}\text { Nutzen Sie im Unterricht regel- } \\
\text { mässig digitale Medien? Und } \\
\text { wenn ja, wie gehen sie hier vor? }\end{array}$ & D2-2 \\
\hline & & $\begin{array}{l}\text { Wie würden Sie Ihren täglichen } \\
\text { Unterricht beschreiben? }\end{array}$ & D3-4 \\
\hline \multirow[t]{3}{*}{$\begin{array}{l}\text { Wie und wo bereiten Sie sich auf } \\
\text { Ihren Unterricht vor? }\end{array}$} & \multirow[t]{3}{*}{ F7 } & $\begin{array}{l}\text { Welche Möglichkeiten zur freien } \\
\text { Unterrichtsvorbereitung haben } \\
\text { Sie? }\end{array}$ & D4-1 \\
\hline & & $\begin{array}{l}\text { Wie kommen Sie mit der verfüg- } \\
\text { baren Zeit zur Unterrichtsvorbe- } \\
\text { reitung aus? }\end{array}$ & D4-6 \\
\hline & & $\begin{array}{l}\text { Wie bereiten Sie Ihre Materialen } \\
\text { für den Unterricht vor? }\end{array}$ & D5-6 \\
\hline \multirow{3}{*}{$\begin{array}{l}\text { Gibt Ihre Schulleitung Regeln vor } \\
\text { oder bietet sie unterstützende } \\
\text { Massnahmen zur Medienintegra- } \\
\text { tion an? }\end{array}$} & \multirow[t]{3}{*}{ F8 } & $\begin{array}{l}\text { Gibt es an Ihrer Schule Regeln } \\
\text { oder Forderungen zum Einsatz } \\
\text { digitaler Medien? }\end{array}$ & D4-2 \\
\hline & & $\begin{array}{l}\text { Wie wirken sich die Forderungen } \\
\text { auf das Kollegium aus? }\end{array}$ & D4-3 \\
\hline & & $\begin{array}{l}\text { Was unternimmt die Schullei- } \\
\text { tung zum Austausch der Lehren- } \\
\text { den untereinander? }\end{array}$ & D4-4 \\
\hline $\begin{array}{l}\text { Welche Debatten finden im Kol- } \\
\text { legium hinsichtlich der Medien- } \\
\text { nutzung statt? }\end{array}$ & F9 & $\begin{array}{l}\text { Welche Debatten finden im Kol- } \\
\text { legium hinsichtlich der Medien- } \\
\text { nutzung statt? }\end{array}$ & D4-5 \\
\hline \multirow[t]{2}{*}{$\begin{array}{l}\text { Welche Äusserungen der Eltern } \\
\text { zu digitalen Medien sind Ihnen } \\
\text { in Erinnerung geblieben und } \\
\text { warum? }\end{array}$} & \multirow[t]{2}{*}{ F11 } & $\begin{array}{l}\text { Welche Äusserungen der Eltern } \\
\text { zu digitalen Medien sind Ihnen } \\
\text { in Erinnerung geblieben und } \\
\text { warum? }\end{array}$ & D6-1 \\
\hline & & $\begin{array}{l}\text { Welche Konsequenzen haben } \\
\text { Sie für sich aus den Äusserungen } \\
\text { gezogen? }\end{array}$ & D6-2 \\
\hline
\end{tabular}




\begin{tabular}{|c|c|c|c|}
\hline Leitfragen & ID-F & Detailfragen & ID-D \\
\hline \multirow[t]{10}{*}{$\begin{array}{l}\text { Welche Erfahrungen haben Sie } \\
\text { beim Einsatz neuer Medien im } \\
\text { Unterricht gemacht? }\end{array}$} & \multirow[t]{10}{*}{ F2 } & $\begin{array}{l}\text { Welche Erfahrungen haben Sie } \\
\text { beim Einsatz digitaler Medien } \\
\text { zum Lernverhalten der Schüler- } \\
\text { innen und Schüler gemacht? }\end{array}$ & D1-1 \\
\hline & & $\begin{array}{l}\text { Welche Erfahrungen haben Sie } \\
\text { beim Einsatz neuer Medien im } \\
\text { Unterricht gemacht? }\end{array}$ & D1-2 \\
\hline & & $\begin{array}{l}\text { Wie schätzen Sie neue Medien } \\
\text { im Vergleich zu herkömmlichen } \\
\text { Medien für den Unterricht ein? }\end{array}$ & D1-4 \\
\hline & & $\begin{array}{l}\text { Was wollen Sie erreichen, wenn } \\
\text { Sie digitale Medien einsetzen? }\end{array}$ & D1-8 \\
\hline & & $\begin{array}{l}\text { Wenn Sie in } 4 \text { Wochen nur noch } \\
\text { digitale Medien im Unterricht } \\
\text { einsetzen dürften, wie würden } \\
\text { Sie vorgehen? }\end{array}$ & D2-1 \\
\hline & & $\begin{array}{l}\text { Wie empfinden Sie das Unter- } \\
\text { richtsgeschehen durch den } \\
\text { Einsatz digitaler Medien im } \\
\text { Vergleich zu herkömmlichen } \\
\text { Medien? }\end{array}$ & D2-3 \\
\hline & & $\begin{array}{l}\text { Wie sehen Sie die Risiken im Ver- } \\
\text { gleich zu den Chancen durch den } \\
\text { Medieneinsatz im Unterricht? }\end{array}$ & D2-4 \\
\hline & & $\begin{array}{l}\text { Inwiefern führt das Chancen-Ri- } \\
\text { siko Verhältnis zur Meidung des } \\
\text { Einsatzes digitaler Medien? }\end{array}$ & D2-5 \\
\hline & & $\begin{array}{l}\text { Beschreiben Sie bitte, wie der } \\
\text { Medieneinsatz in Ihrer Schule } \\
\text { rück-blickend in den vergange- } \\
\text { nen Jahren war? }\end{array}$ & D3-5 \\
\hline & & $\begin{array}{l}\text { Wie empfinden Sie das Unter- } \\
\text { richtsgeschehen durch den Ein- } \\
\text { satz digitaler Medien? }\end{array}$ & D5-5 \\
\hline \multirow[t]{2}{*}{$\begin{array}{l}\text { Welche Eigenschaften würden } \\
\text { Sie digitalen Medien spontan } \\
\text { zurechnen? }\end{array}$} & \multirow[t]{2}{*}{ F3 } & $\begin{array}{l}\text { Welche Eigenschaften würden } \\
\text { Sie digitalen Medien spontan } \\
\text { zurechnen? }\end{array}$ & D1-6 \\
\hline & & $\begin{array}{l}\text { Wie stehen Sie zum Einsatz neu- } \\
\text { er Medien? }\end{array}$ & D3-2 \\
\hline
\end{tabular}




\begin{tabular}{|c|c|c|c|}
\hline Leitfragen & ID-F & Detailfragen & ID-D \\
\hline \multirow[t]{2}{*}{$\begin{array}{l}\text { Wie stehen Sie der Mediennut- } \\
\text { zung der Schülerinnen und Schü- } \\
\text { ler gegenüber? }\end{array}$} & \multirow[t]{2}{*}{ F4 } & $\begin{array}{l}\text { Welche Medien werden nach } \\
\text { Ihrer Einschätzung von den } \\
\text { Schülerinnen und Schüler bevor- } \\
\text { zugt genutzt und warum? }\end{array}$ & D1-5 \\
\hline & & $\begin{array}{l}\text { Wie stehen Sie der Mediennut- } \\
\text { zung der Schülerinnen und Schü- } \\
\text { ler in der Schule gegenüber? }\end{array}$ & D1-7 \\
\hline \multirow{4}{*}{$\begin{array}{l}\text { Welche neuen Medien setzen Sie } \\
\text { privat ein, zu welchen Zwecken } \\
\text { und warum? }\end{array}$} & \multirow[t]{4}{*}{ F6 } & $\begin{array}{l}\text { Wie nehmen Sie die technische } \\
\text { Entwicklung wahr? }\end{array}$ & D5-1 \\
\hline & & $\begin{array}{l}\text { Welche Einstellung haben Sie } \\
\text { gegenüber dem technischen } \\
\text { Wandel? }\end{array}$ & D5-2 \\
\hline & & $\begin{array}{l}\text { Wie schätzen Sie Ihren eigenen } \\
\text { Umgang mit neuen Medien ein? }\end{array}$ & D5-3 \\
\hline & & $\begin{array}{l}\text { Wie und für was nutzen Sie digi- } \\
\text { tale Medien in Ihrem Alltag? }\end{array}$ & D5-4 \\
\hline $\begin{array}{l}\text { Welche aufkommenden Medien } \\
\text { haben Sie in Ihrer Kindheit kon- } \\
\text { sumiert? }\end{array}$ & F5 & $\begin{array}{l}\text { Welche aufkommenden Medien } \\
\text { haben Sie in Ihrer Kindheit kon- } \\
\text { sumiert? }\end{array}$ & D3-1 \\
\hline
\end{tabular}

